\title{
Simulation of the infrared predissociation spectra of $\mathrm{H}_{5}^{+}$
}

\author{
Alfredo Aguado, ${ }^{1}$ Cristina Sanz-Sanz, ${ }^{2}$ Pablo Villarreal, ${ }^{2}$ and Octavio Roncero ${ }^{2, *}$ \\ ${ }^{1}$ Unidad Asociada UAM-CSIC, Departamento de Química Física, Facultad de Ciencias C-XIV, Universidad Autónoma de Madrid, 28049, \\ Madrid, Spain \\ ${ }^{2}$ Unidad Asociada UAM-CSIC, Instituto de Física Fundamental (IFF-CSIC), CSIC, Serrano 123, 28006 Madrid, Spain
}

(Received 14 December 2011; published 16 March 2012)

\begin{abstract}
A quantum study of the bound states and infrared predissociation spectra of $\mathrm{H}_{5}{ }^{+}$is done using a recently proposed global and accurate potential-energy surface [Aguado et al., J. Chem. Phys. 133, 024306 (2010)]. The bound states are calculated for seven degrees of freedom using an iterative Lanczos method, yielding a dissociation energy in very good agreement with the available experimental data. The predissociation states are described by a wave-packet treatment considering a shared-proton model, in which the three-dimensional motion of the central atom is described using non-Jacobi bond coordinates. The justification of this model is that the change in the electric dipole moment is larger as a function of the motion of the central atom, responsible for the proton transfer in the $\mathrm{H}_{3}{ }^{+}+\mathrm{H}_{2} \rightarrow \mathrm{H}_{2}+\mathrm{H}_{3}{ }^{+}$reaction. The electric dipole moment is calculated at the level of coupled-cluster theory with single and double excitations and fitted in nine dimensions to an analytical function. With it, the infrared predissociation spectrum is simulated, yielding a reasonable agreement with recent measurements.
\end{abstract}

DOI: 10.1103/PhysRevA.85.032514

PACS number(s): 33.20.Ea, 87.15.R-, 29.30.Ep

\section{INTRODUCTION}

$\mathrm{H}_{3}{ }^{+}$plays a central role in astrophysics as the most abundant ion in space and as the universal protonator [1-4], so that it is frequently used as a spectroscopic probe of the dynamics of ionospheres of outer planets [5,6]. It is then important to know in detail the rate constants of the collisions in which $\mathrm{H}_{3}^{+}$ participates. Models to estimate the concentration of the cation, as well as its vibrational structure and isotopic substituents, are used to follow the dynamics of the system as a function of solar fluence [7]. In addition, the rates of isotopic exchange processes are important in low-pressure plasmas of $\mathrm{H}_{2}-\mathrm{D}_{2}$ mixtures [8]. The spin statistics of these species determines selection rules for spectroscopy [9-12], and it is therefore also interesting to model the ortho-to-para conversion of $\mathrm{H}_{3}{ }^{+}$and isotopologues $[11,13]$. Particularly intriguing is the relative abundance of deuterated species, which is approximately $10^{4}$ times higher than the D-to-H ratio of the galaxy, [14] attributed to zero-point energy differences important at the low temperatures of the interstellar medium $[15,16]$.

Deuterated $\mathrm{H}_{3}{ }^{+}$is formed via the $\mathrm{HD}+\mathrm{H}_{3}{ }^{+} \rightarrow \mathrm{H}_{2} \mathrm{D}^{+}+$ $\mathrm{H}_{2}$ proton-deuteron exchange reaction, and isotopic variants. These reactions have been studied experimentally [16-21]. There are complementary spectroscopic $\mathrm{H}_{5}^{+}+h v \rightarrow \mathrm{H}_{3}{ }^{+}+$ $\mathrm{H}_{2}$ measurements [22-25] which provide information about the $\mathrm{H}_{5}{ }^{+}$complex structure, energetics, and fragmentation dynamics. These last processes can be viewed as half collisions, allowing a complementary study of the density of states involved in (full) collisions. Of particular relevance is the determination of widths and density of the resonances appearing at low energy, to justify the formation of long-lived complexes supporting or not a statistical description of the reaction.

From the theoretical point of view, there are many studies characterizing this system at fixed geometries [26-34]. Several

*Corresponding author: octavio.roncero@csic.es global potential-energy surfaces (PESs) of increasing accuracy [35-41] have emerged in the last years. An energy diagram for this system is shown in Fig. 1. Since the binding energy is very low, the system becomes very anharmonic and delocalized. In addition, the barrier for the exchange reaction is also below the zero-point energy, so that all identical nuclei can permute. The permutation symmetry constraints imposed by nuclear-spin angular momenta are important, as first treated by Quack [9] and later on by Oka [10], which were experimentally confirmed by measurements in plasmas at 300-500 K [13,42]. All this makes it extremely difficult to perform complete quantum calculations including the twelve degrees of freedom (nine vibrational and three rotational), considering the whole permutation group of symmetry. For this aim, proper coordinates should be designed, like the democratic hyperspherical coordinates developed recently by Kuppermann [43]. The use of these coordinates requires the evaluation of the hyperspherical harmonics, still in development $[44,45]$. Another set of symmetric coordinates was developed to study the spectroscopy of $\mathrm{H}_{5}{ }^{+}$and isotopomers [36,46,47], with respect to an equilibrium geometry. These coordinates were used recently to study the predissociation dynamics [48] using a reduced-dimensional model in an adiabatic approximation, yielding very low predissociation rates.

One of the first simulations of the exchange reaction was the quasiclassical study performed by Moyano and Collins [38], who found discrepancies with the available experimental results, which were attributed to possible quantum effects. Later on, statistical methods, including symmetry constraints, were also applied to these reactions $[12,20]$. The adequacy of these methods relies on the formation of long-lived $\mathrm{H}_{5}{ }^{+}$complexes, which produce a complete randomization of energy among all degrees of freedom. Several models were built assuming the existence or not of a scrambling mechanism, which would allow the exchange of any of the hydrogen atoms within the complex. Only recently have seven-degreeof-freedom quantum scattering calculations of the $\mathrm{H}_{2}+\mathrm{H}_{2} \mathrm{D}^{+}$ 


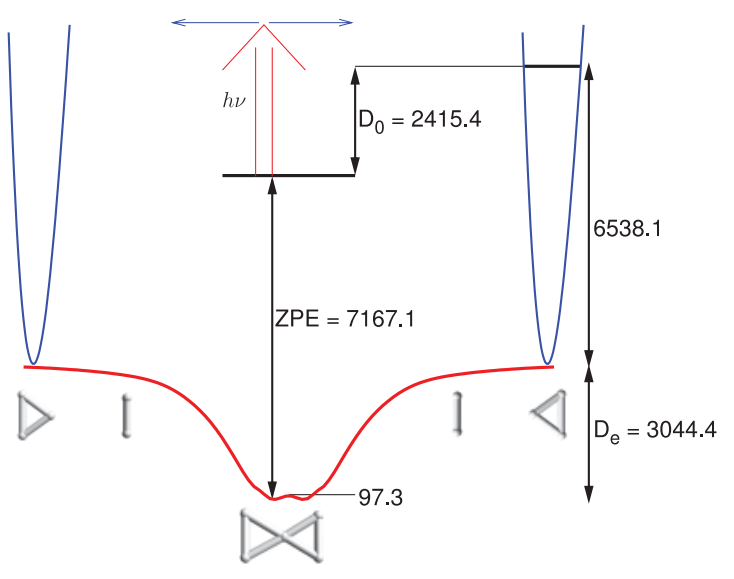

FIG. 1. (Color online) Energy diagram of $\mathrm{H}_{5}{ }^{+}$, obtained with the PES of Ref. [40], using the zero-point energies calculated in this work using the 7D model. Energies are in $\mathrm{cm}^{-1}$.

reaction been reported [49], for state-selected initial states, not taking into account the permutation symmetry. It was found that the reaction occurs without activation energy, showing many resonant structures. The reaction probabilities were not sensitive to the initial vibrational excitation of $\mathrm{H}_{3}{ }^{+}$, but initial rotational excitation can produce an important enhancement of the reaction. The thermal rate coefficients obtained provided a semiquantitative agreement with experimental data. All this demonstrates the need of quantum studies to get deeper insight into the reaction dynamics for this system.

Recently a shared-proton mode was used to describe the infrared predissociation spectra of $\mathrm{H}_{5}^{+}$and $\mathrm{D}_{5}^{+}$[25]. The simulations were done in full dimensions at zero total angular momentum [25], using the reaction path Hamiltonian version of the multimode method [50,51], leading to reasonable but not completely satisfactory agreement with the experimental data. The discrepancies with the experimental results [25] could be attributed to the use of a normal-mode basis set to describe large-amplitude motions, without taking into account the predissociation dynamics of the excited levels reached by infrared absorption. Due to the difficulty of including full dimensions and predissociation dynamics, recently a simple shared-proton model has been developed [52] using two coordinates describing the motion of the central proton between two $\mathrm{H}_{2}$ molecules, providing a reasonable physical assignment of the experimental bands [25]. In this work this simple model is extended to more degrees of freedom, using the same PES [40], with the aim of characterizing the resonances and the main vibrations involved. The electric dipole moment is calculated at coupled-cluster $a b$ initio level and fitted to an analytical function. Calculations include a configuration region which is broad enough to describe the initial bound state. The vibrational levels are calculated for different reduced-dimensional models for zero total angular momentum, with an increasing number of degrees of freedom, up to seven, using an iterative Lanczos method. Finally, the predissociation dynamics is studied using a wave-packet method, characterizing the resonances and simulating infrared predissociation spectra, using a three-dimensional (3D) shared-proton model.
The paper is organized as follows. Section II describes the theoretical methods used to study predissociation dynamics and bound states. Section III describes the calculation and fit of the electric dipole moment. Section IV is devoted to explanation of the vibrational structure as a function of the coordinates, considering several reduced-dimensional models, from two to seven dimensions, and the dissociation energy. In Sec. $\mathrm{V}$ are presented the predissociation dynamics and the spectra, and they are compared to the experimental data. Finally, Sec. VI collects the conclusions of this work.

\section{THEORETICAL METHODOLOGY}

For the study of the vibrational motions and predissociation dynamics of $\mathrm{H}_{5}^{+}$, we consider reduced-dimensional approaches using the coordinates shown in Fig. 2. In these coordinates, a central $\mathrm{H}$ nucleus is at the origin in between two $\mathrm{H}_{2}$ molecules. These coordinates are not Jacobi-like, and there is a kinetic coupling term between $R_{1}$ and $R_{2}$ [53,54]. We define a body-fixed frame with the $z$ axis parallel to the $R_{1}$ vector, and $R_{2}$ lying in the $x-z$ plane. The Hamiltonian for zero total angular momentum, $\mathbf{J}=\mathbf{0}$, can be written as

$$
\begin{aligned}
\hat{H}= & \hat{h}_{1}+\hat{h}_{2}+\hat{T}_{R_{1}}+\hat{T}_{R_{2}}+\left(\frac{\hbar^{2}}{2 \mu_{1} R_{1}^{2}}+\frac{\hbar^{2}}{2 \mu_{2} R_{2}^{2}}\right) \hat{L}^{2} \\
& +\hat{T}_{12}+V,
\end{aligned}
$$

where $V$ is the PES of Ref. [40], $\mu_{i}$ are reduced masses associated with $\boldsymbol{R}_{i}$,

$$
\begin{aligned}
\hat{h}_{i} & =-\frac{\hbar^{2}}{2 m_{i}} \frac{1}{r_{i}^{2}} \frac{\partial}{\partial r_{i}} r_{i}^{2} \frac{\partial}{\partial r_{i}}+\frac{\hbar^{2}}{2 m_{i} r_{i}^{2}} \hat{j}_{i}^{2}, \\
\hat{T}_{R_{i}} & =-\frac{\hbar^{2}}{2 \mu_{i}} \frac{1}{R_{i}^{2}} \frac{\partial}{\partial R_{i}} R_{i}^{2} \frac{\partial}{\partial R_{i}}, \\
\hat{L}^{2} & =-\frac{1}{\sin \gamma} \frac{\partial}{\partial \gamma} \sin \gamma \frac{\partial}{\partial \gamma},
\end{aligned}
$$

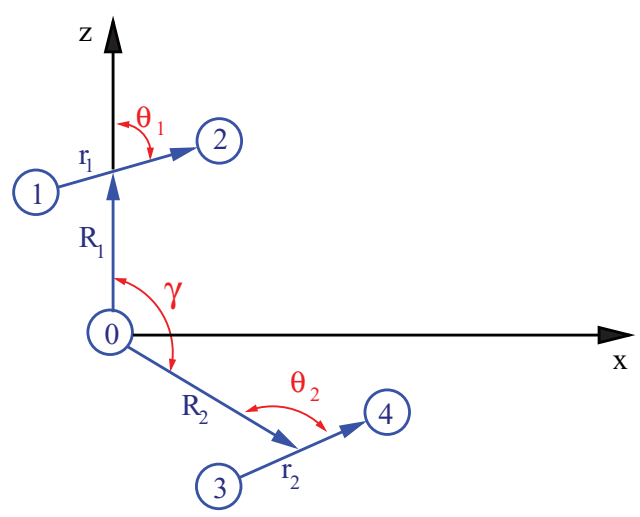

FIG. 2. (Color online) Coordinates used to describe $\mathrm{H}_{5}^{+}$. $\mathbf{r}_{1}$ and $\mathbf{r}_{2}$ are the internuclear vectors between atoms 1 and 2 and 3 and 4 , respectively. $R_{1}$ and $R_{2}$ are the vectors joining atom 0 with the center of mass of diatoms 12 and 34, respectively. $\gamma$ is the angle between $R_{1}$ and $R_{2}$, and $\theta_{i}$ and $\phi_{i}$ are the polar angles of vector $\mathbf{r}_{i}$. For simplicity, in the figure $\phi_{i}$ are set to zero, so that all the atoms are in the $x-z$ body-fixed frame. The global minimum well configuration corresponds to $R_{1}=2.4190$ a.u., $R_{2}=1.6933$ a.u., $r_{1}=1.450$ a.u., $r_{2}=1.532$ a.u., $\gamma=\pi, \theta_{1}=\theta_{2}=\pi / 2$, and $\phi_{1}-\phi_{2}=\pi / 2$. 


$$
\begin{aligned}
\hat{T}_{12}= & \frac{\hbar^{2}}{M_{0}}\left\{\frac{\sin \gamma}{R_{1} R_{2}} \frac{\partial}{\partial \gamma}-\cos \gamma \frac{\partial^{2}}{\partial R_{1} \partial R_{2}}+\frac{\sin \gamma}{R_{2}} \frac{\partial^{2}}{\partial R_{1} \partial \gamma}\right. \\
& \left.+\frac{\sin \gamma}{R_{1}} \frac{\partial^{2}}{\partial R_{2} \partial \gamma}-\frac{\cos \gamma}{R_{1} R_{2}} \hat{L}^{2}\right\},
\end{aligned}
$$

with $m_{i}$ and $\hat{j}_{i}$ being the reduced masses and angular momenta associated with $\mathbf{r}_{i}$, and $M_{0}$ is the mass of the central hydrogen atom. In Eq. (1), it has been assumed that $\mathbf{j}_{1}+\mathbf{j}_{2}=\mathbf{0}$.

The total wave function for $J=0$ is then represented as

$$
\begin{aligned}
& \Psi_{k}^{\Omega}\left(\mathbf{r}_{1}, \mathbf{r}_{2}, R_{1}, R_{2}, \gamma\right) \\
& =\sum_{\ell, v_{1}, v_{2}, v_{1}, v_{2}} \frac{\Phi_{v_{1}, v_{2}, \ell, v_{1}, v_{2}}^{k, \Omega}\left(R_{1}, R_{2}, \theta_{1}, \theta_{2}\right)}{R_{1} R_{2}} \frac{\chi_{v_{1}}\left(r_{1}\right)}{r_{1}} \frac{\chi_{v_{2}}\left(r_{2}\right)}{r_{2}} \\
& \quad \times \mathcal{P}_{\ell \Omega}(\cos \gamma) \frac{e^{-i \nu_{1} \phi_{1}}}{\sqrt{2 \pi}} \frac{e^{-i v_{2} \phi_{2}}}{\sqrt{2 \pi}}
\end{aligned}
$$

where $k$ is the quantum number associated with the $\mathrm{H}_{5}^{+}$ vibrational state, $\theta_{i}, \phi_{i}$ are the angles defining the orientation of $\mathbf{r}_{i}$ as indicated in Fig. 2, with $v_{i}$ being the quantum numbers associated with the angles $\phi_{i}$, and $\mathcal{P}_{\ell \Omega}$ are normalized associated Legendre functions.

Since $\boldsymbol{J}=\mathbf{0}$, its projection $\Omega$ is zero. However, in this work we introduce an ad hoc $\Omega$ with values 0 and \pm 1 , as an approximation to the projection of $\mathbf{J}$ on the body-fixed $z$ axis, to consider parallel $\left(\Omega-\Omega^{\prime}=0\right)$ and perpendicular $\left(\Omega-\Omega^{\prime}=\right.$ $\pm 1)$ transitions. $\chi_{v_{i}}\left(r_{i}\right)$ are vibrational wave functions obtained numerically by solving a one-dimensional radial Schrödinger equation with a well-chosen potential. Typically we use the full potential, where all coordinates, except the one for the study, are frozen at the equilibrium configuration.

Introducing this expansion, Eq. (3), in the time-independent Schrödinger equation yields the following set of coupled differential equations for the $\Phi_{\ell, v_{1}, v_{2}, v_{1}, v_{2}}^{k, \Omega}$ coefficients $[53,54]$ :

$$
\begin{aligned}
\{ & \left.-\frac{\hbar^{2}}{2 \mu_{1}} \frac{\partial^{2}}{\partial R_{1}^{2}}-\frac{\hbar^{2}}{2 \mu_{2}} \frac{\partial^{2}}{\partial R_{2}^{2}}+\left(\frac{\hbar^{2}}{2 \mu_{1} R_{1}}+\frac{\hbar^{2}}{2 \mu_{2} R_{2}}\right) \ell(\ell+1)\right\} \Phi_{\ell, v_{1}, v_{2}, v_{1}, v_{2}}^{k, \Omega}\left(R_{1}, R_{2}, \theta_{1}, \theta_{2}\right)+\sum_{v_{1}^{\prime}} \frac{\hbar^{2}}{2 m_{1}}\left\{\left\langle\chi_{v_{1}}\left|-\frac{\partial^{2}}{\partial r_{1}^{2}}\right| \chi_{v_{1}^{\prime}}\right\rangle\right. \\
& \left.+\left\langle\chi_{v_{1}}\left|\frac{1}{r_{1}^{2}}\right| \chi_{v_{1}^{\prime}}\right\rangle\left\langle v_{1}\left|\hat{j}_{1}^{2}\right| v_{1}\right\rangle\right\} \Phi_{\ell, v_{1}^{\prime}, v_{2}, v_{1}, v_{2}}^{k, \Omega}\left(R_{1}, R_{2}, \theta_{1}, \theta_{2}\right)+\sum_{v_{2}^{\prime}} \frac{\hbar^{2}}{2 m_{2}}\left\{\left\langle\chi_{v_{2}}\left|-\frac{\partial^{2}}{\partial r_{2}^{2}}\right| \chi_{v_{2}^{\prime}}\right\rangle+\left\langle\chi_{v_{2}}\left|\frac{1}{r_{2}^{2}}\right| \chi_{v_{2}^{\prime}}\right\rangle\left\langle v_{2}\left|\hat{j}_{2}^{2}\right| v_{2}\right\rangle\right\} \\
& \times \Phi_{\ell, v_{1}, v_{2}^{\prime}, v_{1}, v_{2}}^{k, \Omega}\left(R_{1}, R_{2}, \theta_{1}, \theta_{2}\right)+\frac{\hbar^{2}}{M_{0}} \sum_{\ell^{\prime}}\left\langle\ell \Omega\left|\hat{t}_{12}\right| \ell^{\prime} \Omega\right\rangle \Phi_{\ell^{\prime}, v_{1}, v_{2}, v_{1}, v_{2}}^{k, \Omega}\left(R_{1}, R_{2}, \theta_{1}, \theta_{2}\right) \\
& +\sum_{\ell^{\prime}, v_{1}^{\prime}, v_{2}^{\prime}, v_{1}^{\prime}, v_{2}^{\prime}}\left\langle\ell, \Omega, v_{1}, v_{2}, v_{1}, v_{2}|V| \ell^{\prime}, \Omega, v_{1}^{\prime}, v_{2}^{\prime}, v_{1}^{\prime}, v_{2}^{\prime}\right\rangle \Phi_{\ell^{\prime}, v_{1}^{\prime}, v_{2}^{\prime}, v_{1}^{\prime}, v_{2}^{\prime}}^{k, \Omega}\left(R_{1}, R_{2}, \theta_{1}, \theta_{2}\right)=E_{k} \Phi_{\ell, v_{1}, v_{2}, v_{1}, v_{2}}^{k, \Omega}\left(R_{1}, R_{2}, \theta_{1}, \theta_{2}\right) .
\end{aligned}
$$

In this expression, the nondiagonal kinetic coupling matrix elements are [54]

$$
\begin{aligned}
\left\langle\ell \Omega\left|\hat{t}_{12}\right| \ell^{\prime} \Omega\right\rangle= & \left\langle\ell \Omega|\cos \gamma| \ell^{\prime} \Omega\right\rangle\left(\frac{1}{R_{1}} \frac{\partial}{\partial R_{2}}+\frac{1}{R_{2}} \frac{\partial}{\partial R_{1}}-\frac{1}{R_{1} R_{2}}-\frac{\partial^{2}}{\partial R_{1} \partial R_{2}}-\frac{\ell^{\prime}\left(\ell^{\prime}+1\right)}{R_{1} R_{2}}\right) \\
& +\left\langle\ell \Omega\left|\sin \gamma \frac{\partial}{\partial \gamma}\right| \ell^{\prime} \Omega\right\rangle\left(\frac{1}{R_{1}} \frac{\partial}{\partial R_{2}}+\frac{1}{R_{2}} \frac{\partial}{\partial R_{1}}-\frac{1}{R_{1} R_{2}}\right),
\end{aligned}
$$

where $\left\langle\ell \Omega|\cos \gamma| \ell^{\prime} \Omega\right\rangle$ and $\left\langle\ell \Omega\left|\sin \gamma \frac{\partial}{\partial \gamma}\right| \ell^{\prime} \Omega\right\rangle$ are given elsewhere $[53,55]$.

The $\Phi_{\ell, v_{1}, v_{2}, v_{1}, v_{2}}^{k, \Omega}\left(R_{1}, R_{2}, \theta_{1}, \theta_{2}\right)$ coefficients are expanded in discrete-variable-representation (DVR) functions. The radial coordinates $R_{i}$ are represented by equidistant points using the DVR basis of Muckerman [56] to evaluate first- and secondorder derivatives. The angular coordinates $\theta_{i}$ are represented in Gauss-Legendre quadrature points and the angular momentum operators are evaluated by a matrix vector multiplication for each $m_{i}$ projection [57-60].

The $\chi_{v}\left(r_{i}\right)$ radial functions are also obtained in a DVR [56] to reduce as much as possible the quadrature points needed. Thus, the potential matrix elements are obtained by numerical integration in the radial variables $r_{1}$ and $r_{2}$. With respect to $\gamma$, an expansion in Legendre polynomials is done as

$$
V\left(\mathbf{r}_{1}, \mathbf{r}_{2}, R_{1}, R_{2}, \gamma\right)=\sum_{\Lambda} \mathcal{V}_{\Lambda}\left(\mathbf{r}_{1}, \mathbf{r}_{2}, R_{1}, R_{2}\right) P_{\Lambda}(\cos \gamma)
$$

and then the integration over $\gamma$ is performed analytically (see Ref. [53], for example) as

$$
\begin{aligned}
& \left\langle\ell \Omega\left|V\left(\mathbf{r}_{1}, \mathbf{r}_{2}, R_{1}, R_{2}, \gamma\right)\right| \ell^{\prime} \Omega^{\prime}\right\rangle \\
& =\sum_{\Lambda} \mathcal{V}_{\Lambda}\left(\mathbf{r}_{1}, \mathbf{r}_{2}, R_{1}, R_{2}\right)\left(\begin{array}{ccc}
\ell & \Lambda & \ell^{\prime} \\
\Omega & 0 & -\Omega^{\prime}
\end{array}\right)\left(\begin{array}{ccc}
\ell & \Lambda & \ell^{\prime} \\
0 & 0 & 0
\end{array}\right) .
\end{aligned}
$$

For $R_{i}$ and $\theta_{i}$ the potential becomes diagonal in DVRs, simplifying the computational effort. For $r_{i}$, the use of basis functions reduces the size of the problem. Thus the potential integrals of the type $\left\langle v_{1}, v_{2}\left|\mathcal{V}_{\Lambda}\left(\mathbf{r}_{1}, \mathbf{r}_{1}, R_{1}, R_{2}\right)\right| v_{1}^{\prime}, v_{2}^{\prime}\right\rangle$ are calculated and stored.

The size of the Hamiltonian matrices thus obtained is too large to be stored and/or to use standard variational diagonalization methods. For this reason an iterative nonorthogonal Lanczos procedure [61] following the method of Cullum and Willoughby [62] has been used to calculate the eigenvalues. 
The Hamiltonian matrix elements are calculated on the fly, without storing them. The eigenstates are obtained iteratively using the conjugate gradient method $[63,64]$ in an efficient way. Due to the high-dimension problem we are dealing with, the size of the matrices is rather big. For this reason the code has been parallelized, in this case on the $r_{i}, \gamma$, and $\theta_{i}$ coordinates.

\section{A. Predissociation spectra}

In the present treatment only zero total angular momentum, $J=0$, is considered. We approximate the electric dipole transition operator simply as the pure electric dipole moment for the ground electronic state of the system, i.e.,

$$
\begin{aligned}
\mathbf{d} \cdot \mathbf{e} & =\sum_{p, q}(-1)^{p} e_{-p} D_{p q}^{1 *}(\alpha, \beta, \phi) d_{q}\left(\mathbf{r}_{1}, \mathbf{r}_{2}, R_{1}, R_{2}, \gamma\right) \\
& \approx \sum_{q} d_{q}\left(\mathbf{r}_{1}, \mathbf{r}_{2}, R_{1}, R_{2}, \gamma\right),
\end{aligned}
$$

where $d_{q}$ is the $q$ projection of the electric dipole moment in the body-fixed frame defined above, and is expanded as

$$
d_{q}\left(\mathbf{r}_{1}, \mathbf{r}_{2}, R_{1}, R_{2}, \gamma\right)=\sum_{\lambda \geqslant|q|} d_{q \lambda}\left(r_{1}, r_{2}, R_{1}, R_{2}\right) \mathcal{P}_{\lambda q}(\gamma) .
$$

In the expression above, $\mathbf{e}$ is the polarization vector of the incident light expressed in a space-fixed reference frame, with projection $e_{p} . D_{p q}^{1 *}(\alpha, \beta, \phi)$ are Wigner rotation matrices [55] which relate the body-fixed and space-fixed frames.

For purely bound-bound transitions, the oscillator strength is then calculated as

$$
I_{k, k^{\prime}} \propto h v \sum_{q}\left|\left\langle\Psi_{k}^{\Omega}\left|d_{q}\right| \Psi_{k^{\prime}}^{\Omega^{\prime}}\right\rangle\right|^{2} .
$$

Since this system is very weakly bound, it is possible to produce its fragmentation into $\mathrm{H}_{2}+\mathrm{H}_{3}{ }^{+}$by infrared transitions. To account for this process, wave-packet calculations are performed using a modified real Chebyshev propagator [65-71]. The initial wave packet is defined as

$$
\Psi_{q, k}^{\Omega^{\prime}}(t=0)=d_{q} \Psi_{k}^{\Omega}
$$

where $\Psi_{k}^{\Omega}$ is the ground bound state of the system, $q=0, \pm 1$, and $\Omega^{\prime}=\Omega+q$. The wave packet is expanded as in Eq. (3), so that the corresponding $\Phi_{v_{1}, v_{2}, \ell, v_{1}, \nu_{2}}^{q k \Omega^{\prime}}\left(R_{1}, R_{2}, \theta_{1}, \theta_{2}, t\right)$ are propagated by solving a linear system of coupled differential equations similar to that written for the bound states in Eq. (4). The main difference is that at each iteration the wave packet is multiplied by an absorption function defined as $e^{-\alpha\left(R_{i}-R_{i}^{\text {abs }}\right)^{2}}$ for $R_{i}>R_{i}^{\text {abs }}$ and 1 elsewhere (with $i=1$ and 2), where $R_{i}^{\text {abs }}$ is the distance in which the wave packet starts being absorbed. The absorption spectrum is then defined as

$$
\begin{aligned}
I_{q k}(E)= & \frac{h v}{\pi \Delta H \sqrt{1-E_{s}^{2}}} \sum_{n}\left(2-\delta_{n 0}\right) \cos \left(-n \arccos E_{s}\right) \\
& \times\left\langle\Psi_{q k}^{\Omega^{\prime}}(n=0) \mid \Psi_{q k}^{\Omega^{\prime}}(n)\right\rangle e^{-n / \tau},
\end{aligned}
$$

where $\Psi_{q k}^{\Omega^{\prime}}(n)$ corresponds to the $n$th Chebyshev iteration $\left[\Psi_{q k}^{\Omega^{\prime}}(n=0)=\Psi_{q k}^{\Omega^{\prime}}(t=0)\right], \quad E_{s}=\left(E-E_{0}\right) / \Delta H$, $E_{0}=\left(E_{\max }+E_{\min }\right) / 2$, and $\Delta H=\left(E_{\max }-E_{\min }\right) / 2$, with $E_{\min }\left(E_{\max }\right)$ being the minimum (maximum) energy of the Hamiltonian represented in the basis set used. The factor $e^{-n / \tau}$ is introduced to eliminate the oscillations appearing from the contribution of bound states after a finite number of iterations. This factor produces an artificial broadening of the bound states and resonances. The broadening is governed by the parameter $\tau$, which depends on the maximum number of iteration considered. This broadening is negligible if sufficiently high $\tau$ and Chebyshev iterations are considered. Here a $\tau$ value of 10 000-20 000 is typically assumed, for 100000 Chebyshev iterations.

The total spectrum, in Eq. (12), gives all the absorption contribution. The bound part of it can be eliminated by projecting out the initial wave packet in the obtained bound states, removing their contribution. This has been done for the lower-dimensional models described below, but for higher dimensions this procedure would involve the calculation of several hundreds of states, which has the problem of the high requirement of CPU time and numerical errors. Instead, we calculate the flux of fragments in the two dissociative channels considered, for a sufficiently long $R_{i}=R_{i}^{\infty}$ distance. This is accomplished, as usual in reactive scattering, as [70]

$$
\begin{aligned}
P_{R}(E) \propto & \sum_{i} \int d \tau_{i} \frac{2 \pi}{\mu_{i} \pi} \\
& \times \operatorname{Im}\left[\left.\Psi_{q k}^{\Omega^{\prime} *}\left(R_{i}^{\infty}, E\right) \frac{d \Psi_{q k}^{\Omega^{\prime}}\left(R_{i}, E\right)}{d R_{i}}\right|_{R_{i}=R_{i}^{\infty}}\right],
\end{aligned}
$$

where it is done for the two coordinates $R_{i}(i=1,2)$ and the integration is performed over all the coordinates except $R_{i}$ and

$$
\begin{aligned}
\Psi_{q k}^{\Omega^{\prime}}(E) \propto & \frac{1}{\pi \Delta H \sqrt{1-E_{s}^{2}}} \sum_{n}\left(2-\delta_{n 0}\right) \\
& \times \cos \left(-n \arccos E_{s}\right) \Psi_{q k}^{\Omega^{\prime}}(n) .
\end{aligned}
$$

These wave functions, at selected energies, have as their main contribution the energy of bound states, which can be used for the assignment of resonances [72-74].

The assignment of different peaks obtained by wave-packet calculations can be done either by comparing with the closest discretized bound state or by performing a pseudospectral analysis of the wave packet at given energies, calculating the wave function at energy $E$ as indicated in Eq. (14).

\section{B. Reduced-dimensional models}

$\mathrm{H}_{5}{ }^{+}$is weakly bound and very anharmonic so large grids and/or basis sets are required to converge the bound states, and even more to converge the wave-packet calculations. It is therefore convenient to perform reduced-dimensional calculations, which, in addition, may help to characterize the vibrational structure of levels and spectra.

The most floppy motion is that associated with $\phi_{1}$ and $\phi_{2}$. In fact, for the equilibrium geometry with $\gamma=\pi$, the system behaves as a diatom + diatom system, and the potential depends only on the $\phi_{1}-\phi_{2}$ difference, as shown in Fig. 3 by freezing all the degrees of freedom except $\phi_{1}$ and $\phi_{2}$. The potential as a function of $\phi_{1}-\phi_{2}$ presents a very shallow well of $90 \mathrm{~cm}^{-1}$ for $\phi_{1}-\phi_{2}=\pi / 2$, below the top of the barrier appearing at $\phi_{1}-\phi_{2}=0, \pi$. The ground monodimensional bound state is only $44 \mathrm{~cm}^{-1}$ above the minimum, and it 


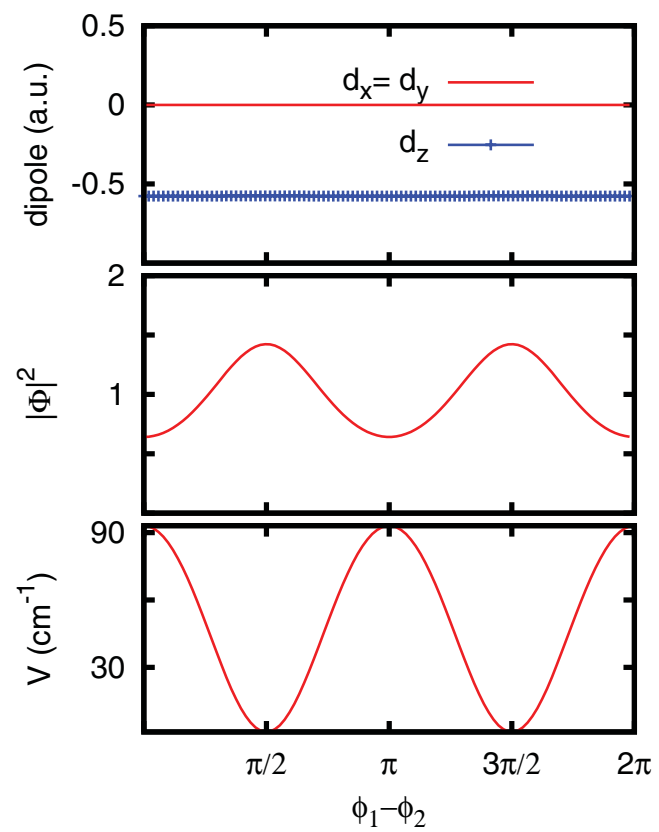

FIG. 3. (Color online) Bottom panel: Potential as a function of $\phi_{1}-\phi_{2}$, at the nuclear configuration of the global minimum with $r_{1}=$ $0.767 \AA, r_{2}=0.8170 \AA, R_{1}=1.27267 \AA, R_{2}=0.895753 \AA, \gamma=$ $\pi, \theta_{1}=\theta_{2}=\pi / 2$. Middle panel: Monodimensional eigenfunction, with energy $44 \mathrm{~cm}^{-1}$. Top panel: Electric dipole moments.

is rather delocalized, as can be seen in Fig. 3. For the equilibrium geometry $\phi_{1}+\phi_{2}$ behaves as a free rotation coordinate, with very low kinetic energy. Thus the inclusion of this mode introduces a high density of states, making the calculation of bound states and resonances extremely complicated. Moreover, the electric dipole moment is rather flat in these two angles, so that infrared excitation will not excite these coordinates, which behave just as spectators. For all these reasons, in the calculations presented below these two angles will be frozen to $\phi_{1}=\phi_{2}=0$, whose motion corresponds to the so-called internal propellerlike rotation in previous studies [36,46,75], where the same approximation was made. To maintain the formalism it will be considered that the potential is isotropic as a function of these two angles and equal to the value of the potential for $\phi_{1}=\phi_{2}=0$.

In what follows the reduced-dimensional models are built as follows:

(a) 2D: $\left(R_{1}, R_{2}\right)$ in which all angles are frozen at their equilibrium values, and $r_{1}, r_{2}$ minimize the potential for each $R_{1}, R_{2}$ distance. This is equivalent to the $2 \mathrm{D}$ treatment used before [52], in which these coordinates were further modified to account for the symmetric and antisymmetric vibrations. Here we keep these coordinates to progressively include more dimensions in the model.

(b) 3D: $\left(R_{1}, R_{2}, \gamma\right)$ as in the previous case but adding the angle $\gamma$ described in a basis of normalized Legendre polynomials.

(c) 4D: $\left(R_{1}, R_{2}, r_{1}, r_{2}\right)$ in which $r_{i}$ are described in a vibrational basis set.

(d) 5D: $\left(R_{1}, R_{2}, r_{1}, r_{2}, \gamma\right)$ as in the previous one but including a Legendre basis set to describe $\gamma$.

(e) 7D: $\left(R_{1}, R_{2}, r_{1}, r_{2}, \theta_{1}, \theta_{2}, \gamma\right)$.
When some coordinate is frozen, only the grid point corresponding to the equilibrium geometry is considered, with the corresponding kinetic-energy operator equal to zero. For the angle $\gamma$, the potential is made isotropic with the value at $\gamma=\pi$ and the kinetic coupling term, in Eq. (5) is approximated by

$$
\left\langle\ell\left|\hat{t}_{12}\right| \ell^{\prime}\right\rangle \approx \frac{\partial^{2}}{\partial R_{1} \partial R_{2}} \delta_{\ell \ell^{\prime}}
$$

\section{ELECTRIC DIPOLE}

The electric dipole moment is needed for the calculation of the spectrum and provides a good description of the charge distribution of $\mathrm{H}_{5}{ }^{+}$. Asymptotically, the $\mathrm{H}_{3}{ }^{+}$fragment presents a $1 / 3$ positive charge on each nucleus. Using a simplistic model, one may think that when $\mathrm{H}_{2}$ approaches $\mathrm{H}_{3}{ }^{+}$, the $\mathrm{H}$ atom in the middle has to take most of the charge in order to produce the proton exchange reaction $\mathrm{H}_{3}{ }^{+}+\mathrm{H}_{2} \rightarrow \mathrm{H}_{2}+\mathrm{H}_{3}{ }^{+}$. As an example, the electronic dipole as a function of $R_{1}$ is shown in Fig. 4, for $r_{1}=r_{2}=1.44$ a.u., $\theta_{1}=\theta_{2}=\pi / 2, \phi_{1}=$ $\phi_{2}=0, \gamma=\pi$, and $R_{2}=6.0-R_{1}$ a.u., and referred to the center of mass of the whole system. At this geometry, only the $z$ component of the dipole moment is nonzero. When the proton is at the same distance between the two $\mathrm{H}_{2}$ molecules, with $R_{1}=3$ a.u., it is also at the center of mass of the system and the $d_{z}$ component becomes zero.

The description of the charge distribution can be done using the diatomics-in-molecules (DIM) and triatomics-inmolecules (TRIM) models [40]. In these methods, an orbital basis set is considered with one $1 s$ function on four of the nuclei and a fifth one with no electron. Five of these orbital functions symmetrically distributed are considered, combined with two singlet electronic spin functions. These models are also used to represent the electric dipole moment, as has already been done for collinear $\mathrm{H}_{3}^{+}$[76]. In the TRIM spin-orbital basis set, the matrix representation of the dipole moment $\mathbf{d}$ is diagonal. This diagonal matrix is transformed to the electronic adiabatic representation using the TRIM eigenvector matrix [76]. The nondiagonal terms of the electric dipole in the final adiabatic representation provide information about the electric dipole moment responsible for electronic transitions, which are not of interest in this work. The diagonal terms provide the electric dipole, in particular that of interest here corresponding to the ground electronic state. The ground TRIM eigenvector provides the weight for each of the ten basis set functions, associated with an electron hole in one of the nuclei, providing a simple way to assign the charge of each hydrogen atom, and thus the electric dipole, as shown in Fig. 4.

The TRIM dipole moment is rather good in the asymptotes, and presents the same behavior as the one from $a b$ initio coupled-cluster theory with single and double excitations (CCSD). In addition, the change of sign, occurring when the central atom 0 is in the neighborhood of the center of mass, is well reproduced. The slope of the TRIM dipole moment is slightly different from the CCSD one, as shown in the bottom panel of Fig. 4. Looking at the charges obtained with the TRIM model, in the top panel of Fig. 4, it is clearly seen that for $R_{1} \approx 1$ a.u. $\left(R_{2}=5\right.$ a.u.), atoms 0,1 , and 2 have the same charges, corresponding to $\mathrm{H}_{3}{ }^{+}$, while for $R_{1} \approx 5$ a.u. 


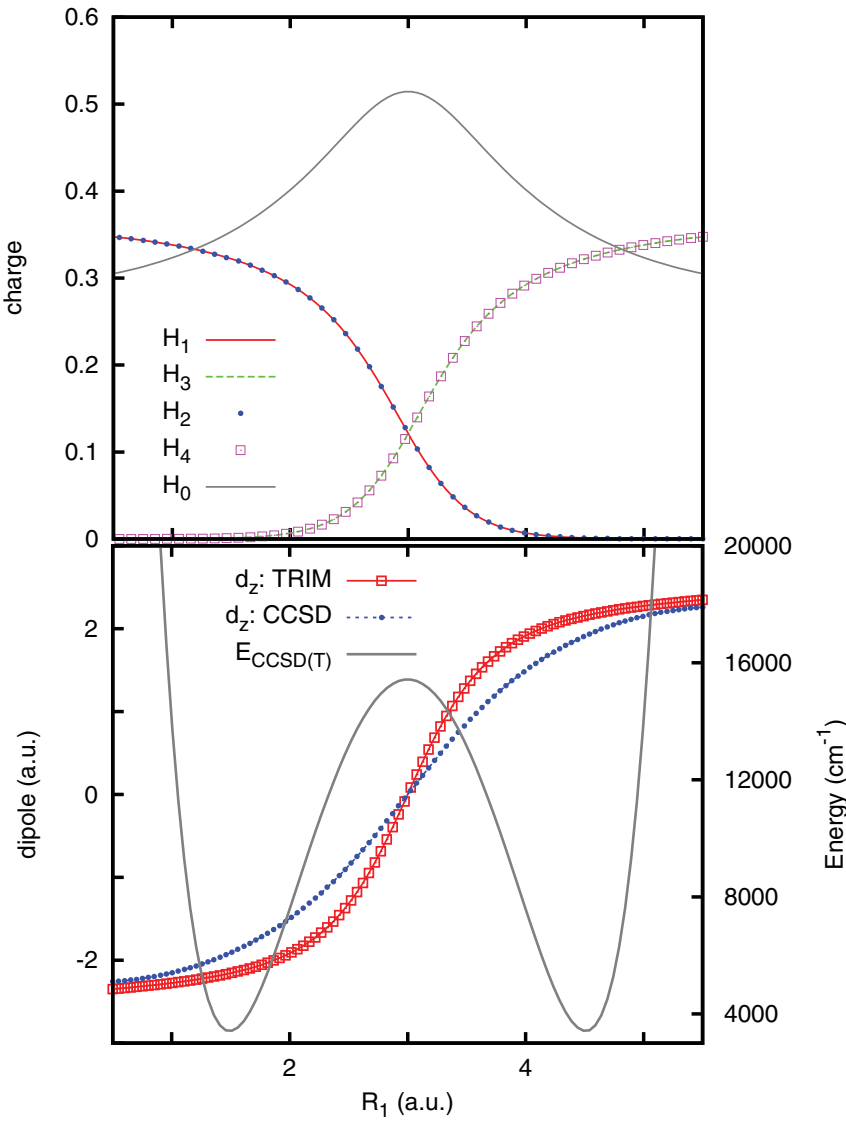

FIG. 4. (Color online) Bottom panel: Electric dipole moment as a function of $R_{1}$ and $R_{2}=6-R_{1}$ a.u. calculated with a CCSD method, compared with that obtained from the TRIM model. The geometry chosen corresponds to $r_{1}=r_{2}=1.4$ a.u., $\theta_{1}=\theta_{2}=\pi / 2, \phi_{1}=\phi_{2}=$ 0 , and $\gamma=\pi$. The corresponding potential energy (in wave numbers) is also shown. Top panel: Charge associated with each of the hydrogen atoms obtained from the TRIM model.

( $R_{2}=1$ a.u.) this charge is transferred to atoms 0,3 , and 4 . In between, the charge is transferred gradually, from atoms $(1,2)$ to atoms $(3,4)$. In fact, it is the atom 0 which increases its charge, and is also the atom transferred. If this reaction is seen as a proton transfer, one may naively think that most of the charge is localized on atom 0 when it occurs. However, in the TRIM model the whole charge is not on the central $\mathrm{H}$ atom at the moment of the proton transfer, but only of the order of 0.52 . The rest of the charge is in the two $\mathrm{H}_{2}$ molecules and is transferred suddenly when the central $\mathrm{H}$ atom "jumps" from one $\mathrm{H}_{2}$ to the other. This is a consequence of the delocalization of the charge. This charge delocalization is not sufficient in the TRIM model, and for this reason the TRIM PES shows a higher barrier between the two $\mathrm{H}_{2}-\mathrm{H}_{3}{ }^{+}$wells [40]. This is the origin of the difference between the TRIM and CCSD models in the description of the electric dipole. Therefore, the TRIM description of the electric dipole should be improved, but keeping its good behavior (topology, change of sign, etc.).

Because the electric dipole moment is a vector, the three components depend on the orientation of the molecule in the Cartesian coordinate system. Furthermore, for charged systems the electric dipole also depends on the origin of the coordinate system. We consider the origin of the coordinate system in the center of mass of the whole system. The fit of the electric dipole is made in the system of the principal axes of inertia of $\mathrm{H}_{5}{ }^{+}$. These axes, labeled as $(a, b, c)$, are chosen so that $I_{a} \leqslant I_{b} \leqslant I_{c}$. Note that the system of principal axes of inertia is only used in the fit, while in the rest of the paper the Cartesian components of the coordinates depicted in Fig. 2 are used.

For the simulation of the spectrum, a total of $100000 \mathrm{ab}$ initio points have been calculated to describe the electric dipole moment. Calculations were done using coupled cluster methods including single and double excitations, as implemented in the MOLPRO package [77], using Dunning's split-valence quadruple- $\zeta$ correlation-consistent basis set (cc-pVQZ) [78]. The grids of the calculations were chosen in a similar way as those used to fit the global PES [40].

The components of the TRIM electric dipole in the principal axes of inertia, $\left(d_{a}, d_{b}\right.$, and $\left.d_{c}\right)$, are presented as functions of the CCSD ones in Fig. 5, showing a strong correlation. In fact the root mean square (rms) deviation of the TRIM dipole with respect to the CCSD one is $0.325,0.096$, and 0.028 a.u for the $d_{a}, d_{b}$, and $d_{c}$ components, respectively. This means that the TRIM model [40] already provides a good qualitative description, and it is used as a starting point.

Nevertheless, the $d_{a}$ component, which accounts for the change of the central proton from reactants $\mathrm{H}_{3}^{+}+\mathrm{H}_{2}$ to products $\mathrm{H}_{2}+\mathrm{H}_{3}{ }^{+}$, has a greater value in the TRIM model than in the CCSD one, probably because the TRIM charge of the central atom is too large. To improve the electric dipole obtained with the TRIM model, we have fitted $d_{a}$ as

$$
d_{a}=d_{a, \mathrm{TRIM}}\left[\alpha-\beta \exp \left(-\gamma d_{a, \mathrm{TRIM}}^{2}\right)\right]
$$

where $\alpha=0.9769, \beta=0.6024$, and $\gamma=0.4254$ a.u. $^{-2}$ are the parameters of the fit. This new fit has the advantage that it is monodimensional, depending only on $d_{a \text {,TRIM }}$ and not on the nine internal coordinates. This procedure reduces the rms error of the $d_{a}$ component, with respect to the CCSD one, to 0.111 a.u, as shown in Fig. 5, improving the good behavior of the TRIM model, in both absolute value and slope. This component is the main one and responsible for the transitions described below. This rms error is similar to those obtained with the TRIM model for the other two components ( $d_{b}$ and $d_{c}$ ), shown in Fig. 5. Other fitting procedures have been checked without significant improvement.

The proton scrambling mechanism corresponds to the rotation of the $\mathrm{H}_{3}{ }^{+}$subunit, so that the central proton changes. In these coordinates this process can be visualized by the simultaneous change of $R_{2}$ and $\gamma$ in a planar configuration, as illustrated in the bottom panel of Fig. 6. The two minima correspond to different central hydrogen atoms, with a barrier lower than $2500 \mathrm{~cm}^{-1}$, which gets lower as the other coordinates are varied. The top panels in Fig. 6 present the nonzero components of the electric dipole, $d_{x}$ and $d_{z}$, and the norm of the dipole vector. The $z$ component changes sign when the angle $\gamma$ goes from $\pi$ (where the central atom is the atom 0 ) to $\pi / 4$ (where the central atom is the atom 2). This implies a sudden change of the direction of the electric dipole vector, but its norm is essentially constant, as shown in the top panel of Fig. 6. 

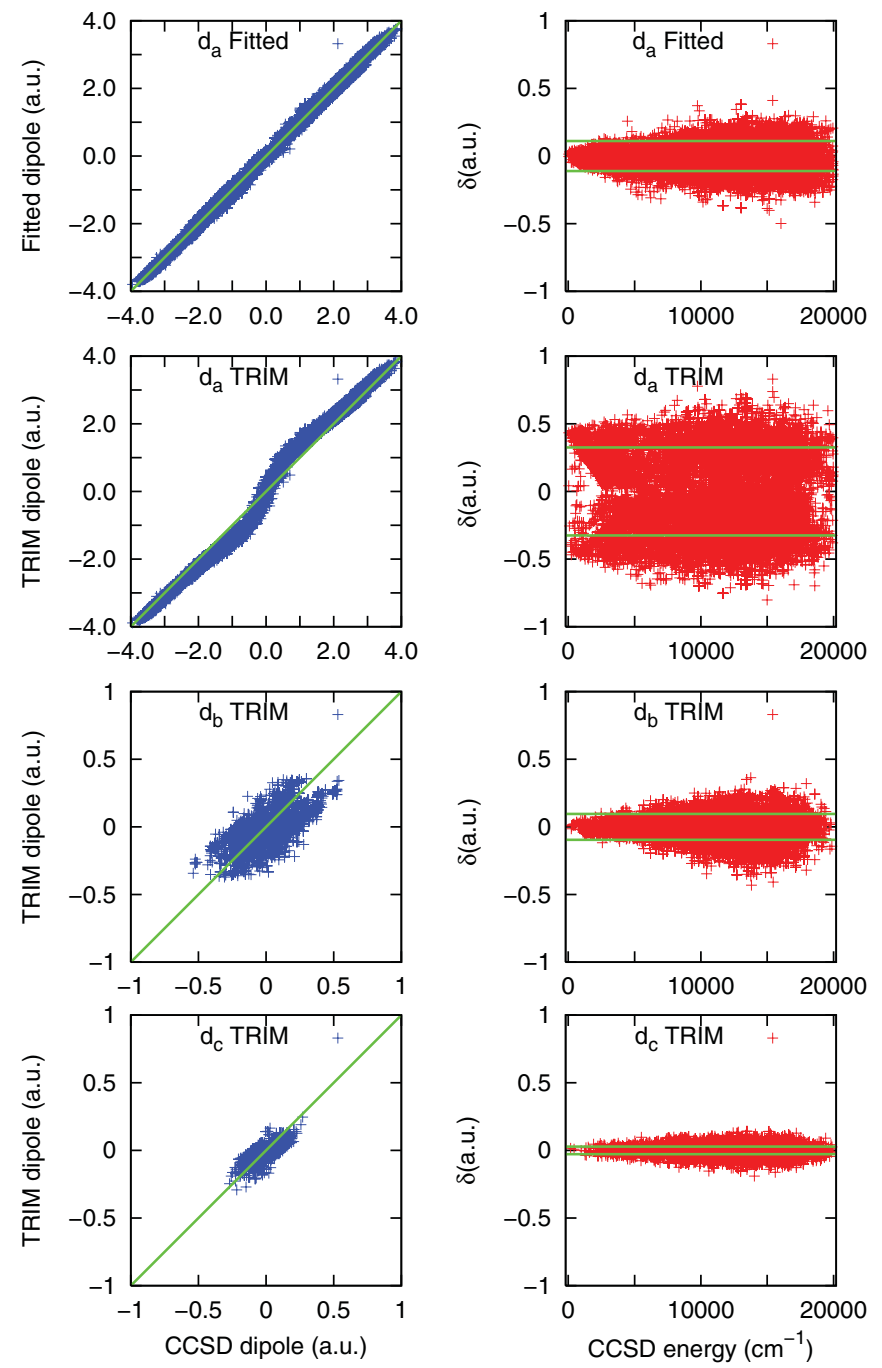

FIG. 5. (Color online) Left panels: Dipole moment components $d_{a}, d_{b}$, and $d_{c}$ obtained with the TRIM (three lower panels) and TRIM + fit (top panel) models as a function of the exact CCSD one. Right panels: Residuals of the dipole moment components $d_{a}, d_{b}$, and $d_{c}$ as functions of the total energy for the TRIM model (three lower panels) and TRIM + fit $d_{a}$ (top panel). Green solid lines correspond to the rms error.

\section{BOUND STATES AND DISSOCIATION ENERGY}

The zero-point energy (ZPE) of the $\mathrm{H}_{3}{ }^{+}$and $\mathrm{H}_{2}$ fragments are calculated using the global nine-dimensional PES [40] of $\mathrm{H}_{5}{ }^{+}$at a distance of 100 a.u. between the two fragments. For $\mathrm{H}_{2}$ the rovibrational states are calculated numerically by solving the monodimensional radial Schrödinger equation. The ground vibrational states are $2178.6 \mathrm{~cm}^{-1}$ for para-hydrogen $(j=0)$ and 2296.83 for ortho-hydrogen $(j=1)$. The eigenvalues of the $\mathrm{H}_{3}{ }^{+}$fragment have been calculated using hyperspherical coordinates for a total angular momentum $\mathbf{J}=\mathbf{0}$. The method used for the calculation of these vibrational states is already described on Refs. [79,80]; the values obtained are listed in Table I. The vibrational levels are compared with those obtained for $\mathrm{H}_{3}{ }^{+}$exclusively, which are of higher accuracy. The comparison is excellent for the lower vibrational states, with an energy difference of simply $1-2 \mathrm{~cm}^{-1}$. These differences

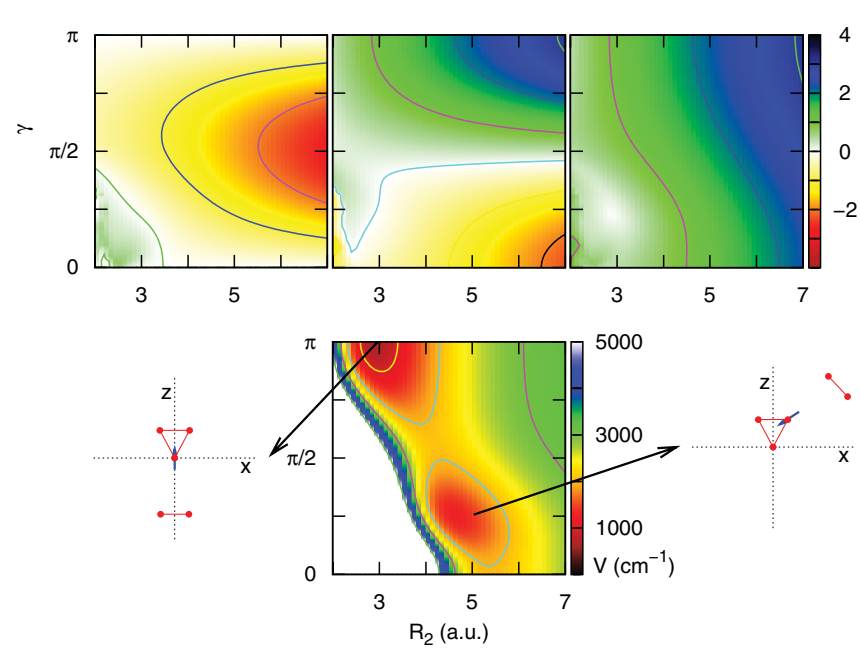

FIG. 6. (Color online) Bottom panel: PES as a function of $R_{2}$ and $\gamma$, for $r_{1}=1.5325$ a.u., $r_{2}=1.4499$ a.u., and $\theta_{1}=\theta_{2}=\pi / 2$, $\phi_{1}=\phi_{2}=0$, corresponding to their values at the minimum of the PES, while $R_{1}=1.4288$ a.u. has been slightly reduced to make the corresponding $\mathrm{H}_{3}{ }^{+}$closer to an equilateral triangle. The two minima correspond to different central hydrogen atoms, as example of the scrambling motion in these coordinates, with a barrier below $2500 \mathrm{~cm}^{-1}$. In the two insets indicating the geometry, the vector represents the electric dipole moment. The contours are 0, 1000, $2000 \mathrm{~cm}^{-1}$, etc. Top panels: Fit of the electric dipole in a.u., $d_{x}$ (left) and $d_{z}$ (middle) components and norm (right) of the $\mathbf{d}$ vector. The contours are $-3,-2,-1$ a.u., etc.

increase with energy, so that for vibrational levels $10000 \mathrm{~cm}^{-1}$ above the ground state the error becomes of the order of $8 \mathrm{~cm}^{-1}$. This check demonstrates the accuracy of the PES [40] to describe the fragments even at quite high vibrational excitations.

The $A_{1}$ states of $\mathrm{H}_{3}{ }^{+}$do not exist, because of the permutation symmetry. Only $A_{2}$ (ortho- $\mathrm{H}_{3}{ }^{+}$with nuclear spin $I=3 / 2$ ) and $E$ (para- $\mathrm{H}_{3}{ }^{+}, I=1 / 2$ ) states exist. Therefore, the ground state is a $\left(0,0^{0}\right)$ vibrational state but with total angular momentum $J=1$ of $E$ symmetry, with an energy of $64 \mathrm{~cm}^{-1}$ above the $\left(0,0^{0}\right)$ level of $\mathrm{H}_{3}^{+}$with $J=0$ and $A_{1}$ symmetry. This energy difference is small, comparable with the accuracy of the reduced-dimensional models presented below, and smaller than the contribution of the $\phi_{i}$ angles to the zero-point energy. In fact, the lower transition bands of $\mathrm{H}_{3}^{+}, \nu_{2}\left(0,1^{1}\right) \leftarrow\left(0,0^{0}\right)$ and $v_{1}\left(1,0^{0}\right) \leftarrow\left(0,0^{0}\right)$ are characterized by an energy very close to the corresponding energies listed in Table I for $J=0$.

In the present model the symmetry is not taken into account, so $\mathrm{H}_{5}{ }^{+}$can fragment, producing $\mathrm{H}_{3}^{+}$in its ground vibrational state $\left(0,0^{0}\right)$, with $J=0$ and $A_{1}$ symmetry. This assumption introduces an error of the order of $60 \mathrm{~cm}^{-1}$ in the threshold energy. Nevertheless, to be consistent, here we will consider a ZPE of fragments of $4359.5+2178.6=6538.1 \mathrm{~cm}^{-1}$, which is at $9582.5 \mathrm{~cm}^{-1}$ above the bottom of the well; see Fig. 1.

The bound states of $\mathrm{H}_{5}^{+}$have been calculated for an increasing number of coordinates, to analyze gradually their ZPE contribution, frequency, and structure. The ZPE and first eigenvalues obtained are listed in Table II. For the 2D $\left(R_{1}, R_{2}\right)$ and 3D $\left(R_{1}, R_{2}, \gamma\right)$ cases, the potential corresponds to the $\left(r_{1}, r_{2}\right)$ couple of lower energy, keeping the remaining angles 
TABLE I. Vibrational energies of $\mathrm{H}_{3}^{+}$and zero total angular momentum, referred to the ground vibrational state. The zero-point energies are also listed in parentheses.

\begin{tabular}{|c|c|c|c|c|c|c|c|}
\hline$i(\Gamma, J)$ & $\left(v_{1}, v_{2}^{\ell}\right)$ & $\Gamma$ & $\mathrm{H}_{3}{ }^{+}$in $\mathrm{H}_{5}^{+}$ & PES of Ref. [79] & PES of Ref. [88] & PES of Ref. [89] & Expt. + fit [90] \\
\hline 1 & $\left(0,0^{0}\right)$ & $A_{1}^{\prime}$ & $0.0(4359.5)$ & 0.0 & 0.0000 & 0.0000 & 0.0000 \\
\hline 2 & $\left(1,0^{0}\right)$ & $A_{1}^{\prime}$ & 3178.2 & 3179.0519 & 3178.7480 & 3178.15 & \\
\hline 3 & $\left(0,2^{0}\right)$ & $A_{1}^{\prime}$ & 4772.9 & 4777.5859 & 4777.1021 & 4778.01 & \\
\hline 4 & $\left(2,0^{0}\right)$ & $A_{1}^{\prime}$ & 6261.9 & 6263.5199 & 6260.4428 & 6261.81 & \\
\hline 5 & $\left(0,3^{3}\right)$ & $A_{1}^{\prime}$ & 7277.0 & 7284.4403 & 7283.1507 & 7285.32 & \\
\hline 6 & $\left(1,2^{0}\right)$ & $A_{1}^{\prime}$ & 7763.5 & 7769.2189 & 7766.7933 & 7768.84 & \\
\hline 7 & $\left(0,4^{0}\right)$ & $A_{1}^{\prime}$ & 8990.8 & 8999.7805 & 8995.3972 & 9001.36 & \\
\hline 8 & $\left(3,0^{0}\right)$ & $A_{1}^{\prime}$ & 9251.8 & 9253.8739 & 9245.7749 & 9251.42 & \\
\hline 9 & $\left(1,3^{3}\right)$ & $A_{1}^{\prime}$ & 9959.6 & 9967.7915 & 9963.1895 & 9968.34 & \\
\hline 1 & $\left(0,3^{3}\right)$ & $A_{2}^{\prime}$ & 7485.5 & 7492.3924 & 7491.4193 & 7492.61 & 7493.113 \\
\hline 2 & $\left(1,3^{3}\right)$ & $A_{2}^{\prime}$ & 10202.5 & 10209.4802 & 10206.0906 & 10209.65 & \\
\hline 1 & $\left(0,1^{1}\right)$ & $E^{\prime}$ & 2518.8 & 2521.0691 & 2520.9840 & 2521.20 & 2521.422 \\
\hline 3 & $\left(0,2^{2}\right)$ & $E^{\prime}$ & 4992.8 & 4997.5038 & 4996.9634 & 4997.73 & 4998.058 \\
\hline 5 & $\left(1,1^{1}\right)$ & $E^{\prime}$ & 5550.8 & 5554.5537 & 5554.2073 & 5553.95 & 5554.274 \\
\hline 7 & $\left(0,3^{1}\right)$ & $E^{\prime}$ & 6997.9 & 7004.9340 & 7003.2497 & 7005.81 & 7006.187 \\
\hline 9 & $\left(1,2^{2}\right)$ & $E^{\prime}$ & 7864.2 & 7869.9785 & 7868.5633 & 7869.09 & 7870.664 \\
\hline 11 & $\left(2,1^{1}\right)$ & $E^{\prime}$ & 8484.8 & 8488.8108 & 8485.4081 & 8487.53 & \\
\hline 13 & $\left(0,4^{2}\right)$ & $E^{\prime}$ & 9101.7 & 9111.0776 & 9107.2931 & 9112.90 & \\
\hline 15 & $\left(1,3^{1}\right)$ & $E^{\prime}$ & 9643.7 & 9652.4868 & 9649.7530 & 9653.44 & \\
\hline 17 & $\left(0,4^{4}\right)$ & $E^{\prime}$ & 9988.9 & 9996.8834 & 9990.8078 & 9996.67 & \\
\hline
\end{tabular}

at their equilibrium values $\left(\gamma=\pi, \theta_{1}=\theta_{2}=\pi / 2\right)$. The ZPE increases gradually with the inclusion of more degrees of freedom, and assuming an approximately additive contribution for each coordinate to the ZPE, we can estimate the ZPE associated with each of them. Thus, the larger frequency is associated with $r_{1}, r_{2}$ stretching motions, with a ZPE of $\approx 1932-1997 \mathrm{~cm}^{-1}$, i.e., smaller than that of free $\mathrm{H}_{2}$ by only $200 \mathrm{~cm}^{-1}$. These two vibrations are not very much affected within the $\mathrm{H}_{5}^{+}$complex. The ZPE associated with each $R_{i}$ is $\approx 544 \mathrm{~cm}^{-1}$. The vibrations of $R_{1}$ and $R_{2}$ coordinates are strongly coupled. The ZPE of $\gamma$ is $\approx 1070 \mathrm{~cm}^{-1}$, while that for each $\theta_{i}$ is $574 \mathrm{~cm}^{-1}$.

All the ZPEs given before consider a planar configuration where $\phi_{1}=\phi_{2}=0$, with an energy of $\approx 90 \mathrm{~cm}^{-1}$ above the global minimum. When the $\phi_{1}$ and $\phi_{2}$ are included [see the last column of Table II, $\left.5 \mathrm{D}\left(\gamma, \theta_{1}, \theta_{2}, \phi_{1}, \phi_{2}\right)\right]$ the ZPE becomes $80 \mathrm{~cm}^{-1}$ lower than that for the $3 \mathrm{D}\left(\gamma, \theta_{1}, \theta_{2}\right)$ case. If this effect is considered in the 7D model, the ZPE can vary by $\approx 60-80 \mathrm{~cm}^{-1}$.

Using the 7D ZPE, of $7167.1 \mathrm{~cm}^{-1}$ (see Table II), and the energy of the fragments in their ground vibrational state described above, $9582.5 \mathrm{~cm}^{-1}$, the dissociation energy of the system is $D_{0}=2415.4 \mathrm{~cm}^{-1}$, with an uncertainty of $\approx 100 \mathrm{~cm}^{-1}$ due to the ZPE contribution of the $\phi_{i}$ angles and the symmetry and rotation problem already mentioned. This value is in agreement with those calculated previously using different PESs of 2455.3 [81], $\leqslant 2602$ [25], 2227.9 [82], and 2213.9 [39]. In addition, this value is also in good agreement with the available experimental dissociation energies: $2308 \pm 105$ [83], $2413 \pm$ 104 [84], and $2448 \pm 35 \mathrm{~cm}^{-1}$ [85]. We conclude that the dissociation energy in the present 7D model, using the PES of Ref. [40], is in good agreement with the values in the literature.

$\mathrm{H}_{5}{ }^{+}$presents bound states that are very delocalized and anharmonic, which complicates the calculation of vibrational

TABLE II. First vibrational eigenvalues for different reduced-dimensional models, each referred to its own ground level. The zero-point energy is given in parentheses. All energies are in $\mathrm{cm}^{-1}$. The first column corresponds to the results shown in Table II of Ref. [75]. The parameters used in the calculations are 90 points in $0.6 \leqslant R_{i} \leqslant 16$ a.u., 6 vibrational functions for $r_{i}$, 30 Legendre polynomials for $\gamma$, and 30 Gauss-Legendre points for $\theta_{i}$. For the 7D case, the number of points in $R_{i}$ has been reduced to 60, and for $\theta_{i}$ to 15 , and the number of vibrational functions on $r_{i}$ to 4 , so that the full Hamiltonian matrix has a dimension of $\approx 4 \times 10^{8}$.

\begin{tabular}{|c|c|c|c|c|c|c|c|}
\hline Ref. [75] & $7 \mathrm{D}$ & $\begin{array}{c}5 \mathrm{D} \\
\left(r_{1}, r_{2}, R_{1}, R_{2}, \gamma\right)\end{array}$ & $\begin{array}{c}4 \mathrm{D} \\
\left(r_{1}, r_{2}, R_{1}, R_{2}\right)\end{array}$ & $\begin{array}{c}3 \mathrm{D} \\
\left(R_{1}, R_{2}, \gamma\right)\end{array}$ & $\begin{array}{c}2 \mathrm{D} \\
\left(R_{1}, R_{2}\right)\end{array}$ & $\begin{array}{c}3 \mathrm{D} \\
\left(\gamma, \theta_{1}, \theta_{2}\right)\end{array}$ & $\begin{array}{c}5 \mathrm{D} \\
\left(\gamma, \theta_{1}, \theta_{2}, \phi_{1}, \phi_{2}\right)\end{array}$ \\
\hline (7413) & $(7167.1)$ & (6019.9) & (4953.2) & (2159.3) & (1088.1) & (2187.9) & (2107.4) \\
\hline 302 & 255.5 & 183.2 & & 306.8 & & & \\
\hline 736 & 636.2 & 541.5 & 474.7 & 522.0 & 533.2 & & \\
\hline 813 & 763.7 & 693.2 & & 602.1 & & & \\
\hline 1006 & 889.0 & 839.8 & 817.6 & 867.5 & 871.6 & & \\
\hline 1102 & 1047.0 & 1005.0 & 1178.0 & 994.0 & 1259.5 & & \\
\hline
\end{tabular}


states. In fact, the ZPE is above the asymptotic potential-energy value, what makes the wave functions very delocalized. The harmonic approximation does not work well in this situation: the calculated harmonic ZPE is $7652 \mathrm{~cm}^{-1}$, higher than the one reported here, $7167 \mathrm{~cm}^{-1}$, which includes anharmonic effects. The analysis of the vibrations is done by comparing the first vibrational levels for different reduced-dimensionality models, referred to the ground-state vibrational level, listed in Table II. The 7D eigenvalues present a progression close to those reported previously using another PES and symmetric coordinates [75]. There are deviations of the order of $100 \mathrm{~cm}^{-1}$, which is considered satisfactory, since the potentials are different, and the difference between the ZPE values is $\approx 250 \mathrm{~cm}^{-1}$. Because of the anharmonicity of the system the energies are very strongly dependent on the coordinates used, especially when any of them is frozen. The close analogy between the vibrational progressions obtained in the $3 \mathrm{D}, 5 \mathrm{D}$, and $7 \mathrm{D}$ models indicates that the 3D model already catches some of the most important ingredients of the spectrum. These dimensions correspond to a proton-shared model [52], with a central proton vibrating in between the two $\mathrm{H}_{2}$ subunits, producing changes in the electric dipole responsible for the transitions.

For the analysis of bound states we use two sets of coordinates, those used for the calculations and introduced in Fig. 2, and hyperspherical coordinates. These last coordinates are defined as $\rho=\sqrt{R_{1}^{2}+R_{2}^{2}}$ and $\xi=\arctan \left(R_{1} / R_{2}\right)$, and are directly associated with the symmetric and antisymmetric stretching vibrations $(s, a)$, related to the coordinates $S_{1}\left(A_{1}^{+}\right)$ and $S_{3}\left(B_{2}^{+}\right)$defined in Refs. [36,46,48]. These hyperspherical coordinates are appropriate for the assignment of the bound states, as are those used before in the 2D model of Ref. [52], leading to a rather similar description. Thus, the amplitude densities for the five first 3D vibrational states, shown in Fig. 7, are assigned to a given $(s, a, b)$ set of quantum numbers. $s$ and $a$ correspond to the symmetric and antisymmetric stretching motions, and $b$ refers to bending motion associated with $\gamma$. These levels present a clear separation between even and odd $a$ values, with a central node at $\xi=\pi / 4$.

The first excited state corresponds to an excitation of the antisymmetric stretch, $a=1$, vibration which is directly related to dissociation. The second and third excited bound states (marked with asterisks in the figure) are already strongly mixed. They can be approximately assigned to the first excitation of the symmetric mode, $s=1$, and the first excitation of the bending mode, $b=1$. These two bound states present a large amplitude for $R_{2}=5$ a.u. and $\gamma=\pi / 6$, a configuration which corresponds to one of the minima in the potential plotted on Fig. 6. This minimum is assigned to the configuration where the central atom is 2 instead of 0 . The amplitude is very delocalized in the $\gamma$ coordinate, showing that at only $500-600 \mathrm{~cm}^{-1}$ the $\mathrm{H}_{3}^{+}$subunit can rotate within the whole complex. This allows any one of the $\mathrm{H} \mathrm{of}_{3}{ }^{+}$to be the central atom. This motion is associated with the scrambling mechanism [20] in collisions, in which any $\mathrm{H}$ atom can be exchanged. Finally, the fifth bound state is assigned to an excitation of the symmetric and antisymmetric stretches. We can conclude that the lower-frequency vibrations correspond to the motion of the central atom. The motions of the two $\mathrm{H}_{2}$

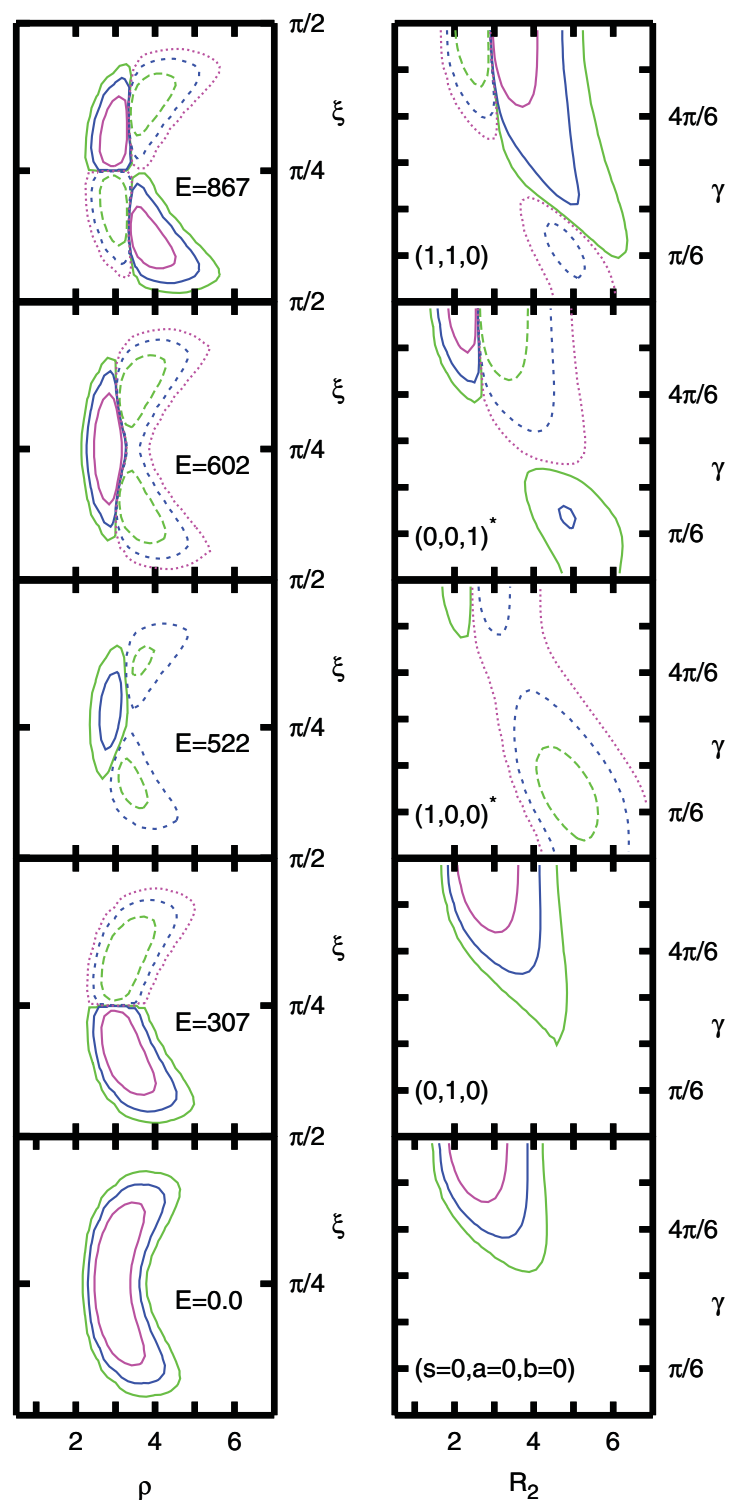

FIG. 7. (Color online) Contour plots of the wave functions of the first five 3D levels shown in Table II, as a function of two different coordinates. Left panels: As a function of $\rho=\sqrt{R_{1}^{2}+R_{2}^{2}}$ and $\xi=$ $\arctan \left(R_{1} / R_{2}\right)$ keeping $\gamma=\pi$. Right panels: As a function of $R_{2}$ and $\gamma$ keeping $R_{1}=1.63$ a.u. Contours are $1,0.1,0.001$,etc. The energy of each level $\left(\right.$ in $\mathrm{cm}^{-1}$ ) is in the left panels, with respect to the minimum $\mathrm{ZPE}$ of the $3 \mathrm{D}$ model. Distances are in a.u. and angles in radians.

units are of higher frequency, similar to those of free $\mathrm{H}_{2}$, and have amplitudes which do not change appreciably.

The vibrations associated with $R_{i}$ within $\mathrm{H}_{3}{ }^{+}$in the $\mathrm{H}_{2}+$ $\mathrm{H}_{3}{ }^{+}$asymptote are of much higher frequency. The symmetric and antisymmetric vibration frequencies in $\mathrm{H}_{3}^{+}$are $v_{1} \approx$ $3178 \mathrm{~cm}^{-1}$ and $\nu_{2} \approx 2518 \mathrm{~cm}^{-1}$ [see the $\left(0,0^{0}\right)$ and $\left(0,1^{1}\right)$ levels in Table I]. When the $\mathrm{H}_{2}$ fragment approaches $\mathrm{H}_{3}{ }^{+}$, the proton is delocalized between the two $\mathrm{H}_{2}$ subunits with a much lower frequency, decoupled from the two $\mathrm{H}_{2}$ stretching motions. Thus, the $\mathrm{H}_{2}$ distances increase when the proton approaches them; the $\mathrm{H}-\mathrm{H}$ distance is slightly longer in $\mathrm{H}_{3}{ }^{+}$ than in $\mathrm{H}_{2}$. In this situation, the two stretching motions along $R_{1}$ and $R_{2}$ become coupled. 
When an infrared transition is considered, all the oscillator strength goes to excite the antisymmetric mode $a$, because the electric dipole moment is also antisymmetric. The transitions toward $a=1$ are the most intense ones, as shown in Fig. 3 of Ref. [25]. As an example, the intensity factors $I_{k=0, k^{\prime}}$, in Eq. (10), have been calculated in the 3D model, corresponding to transitions from the ground state to the $k^{\prime}>0$ bound states shown in Fig. 7. The values obtained are $0.4,4 \times 10^{-6}$, $2 \times 10^{-5}$, and $8 \times 10^{-3}$ to $k^{\prime}=1,2,3$, and 4 , respectively. The first transition is by far the most intense and takes about $40 \%$ of the oscillator strength. It corresponds to the $(0,0,0) \rightarrow(0,1,0)$ transition in which the antisymmetric mode is excited by one quantum. The following two intensity factors correspond to symmetric bound states, $(1,0,0)$ and $(0,0,1)$, and they should be zero. They are not because of numerical errors in the integrals when using nonsymmetric coordinates. Finally, the fourth factor corresponds to a transition to the $(1,1,0)$ odd bound state with one excitation quantum in the symmetric stretch, which accounts for only $0.8 \%$ of the oscillator strength. There are many other bound states below the dissociation limit but they are difficult to converge. In the following, we study the transitions corresponding to resonances or quasibound states lying above the dissociation limit.

\section{PREDISSOCIATION RATES AND INFRARED SPECTRA}

\section{A. Two-dimensional infrared spectrum}

The relatively shallow well, of $\approx 3044 \mathrm{~cm}^{-1}$, supports only a few bound states, and when the system is excited with infrared radiation, the $\mathrm{H}_{5}^{+}$complex dissociates into $\mathrm{H}_{3}^{+}+\mathrm{H}_{2}$ fragments, as has been already measured in several laboratories [22-25]. The $\mathrm{H}_{2}+\mathrm{H}_{3}{ }^{+}$fragmentation channels are associated with the lengthening of $R_{1}$ or $R_{2}$. We first study the two-dimensional $R_{1}, R_{2}$, case in which the angular variables are frozen to their equilibrium values, $\theta_{1}=\theta_{2}=\pi / 2$ and $\gamma=\pi$ (with $\phi_{1}=\phi_{2}=0$ ), while $r_{1}$ and $r_{2}$ are varied as functions of $R_{1}$ and $R_{2}$ to get the minimum of the PES for each $R_{1}, R_{2}$ couple. This model correspond to the 2D model used before [52], in which an adiabatic separation of these two motions was done, while here they are treated exactly. Thus, the first bound states have very close energy and the same vibrational structure. As the energy increases the difference increases, but most of the results obtained are in rather good qualitative agreement with those obtained in Ref. [52].

The grids used here to describe $R_{1}$ and $R_{2}$ are composed of 180 points in the intervals $0.2 \leqslant R_{i} \leqslant 30$ a.u. The first bound state is calculated using a Lanczos procedure, as described in the previous section, and then is multiplied by the electric dipole moment already described, using the approximation of Eq. (11). The initial wave packet is propagated using a Chebyshev method, so that the infrared spectrum is obtained from the autocorrelation function Eq. (12), and from the analysis of the fluxes at $R_{i}^{\infty}=21$ a.u., using Eq. (13). These two spectra are shown in Fig. 8, and they coincide for energies above the dissociation threshold, at $3379.6 \mathrm{~cm}^{-1}$ above the ground 2D state (see Table II) and marked with an arrow in the figure. Below this threshold the spectrum corresponds to bound-bound transitions, similar to that reported previously for another potential [25]. These spectra are artificially broadened by the inclusion of the exponential damping in Eq. (12), with $N_{\text {max }}=10^{5}$, to avoid infinite propagations. Also, the wave packet is absorbed at the edges of the grid by multiplying the wave packet at each iteration by an adsorption potential defined as $e^{-0.001\left(R_{i}-R_{i}^{\text {abs }}\right)^{2}}$ for $R_{i}>R_{i}^{\mathrm{abs}}=22$ a.u. The peaks calculated show a natural broadening associated with vibrational predissociation, and convergence is demonstrated by the good agreement obtained in the spectra calculated with the two different methods, shown in Fig. 8(a) (above $8000 \mathrm{~cm}^{-1}$ the flux probability was not analyzed). The same results were obtained for a smaller grid composed of $90 \times 90$ points in the interval $0.6 \leqslant R_{i} \leqslant 16$ a.u. ( $R_{i}^{\text {abs }}=12$ a.u.). This smaller grid is used for higher-dimensional models.

Some of the more relevant peaks have been fitted to a Lorentzian function to extract their half widths at half maximum $\Gamma$, which are shown in Fig. 8(a). The calculated widths are of the order of $5-80 \mathrm{~cm}^{-1}$, corresponding to lifetimes of the order of 1-70 fs. Some broadening is due to the absorption of the wave packet at the edges of the grids, whose contribution is estimated to be of the order of $1 \mathrm{~cm}^{-1}$ or less from the widths obtained for the bound states below the dissociation limit. These widths are considerably larger than those reported previously [48]. The authors of that reference used symmetric coordinates and an adiabatic approximation together with a stabilization method. Predissociation rates are very sensitive to the decoupling scheme, which depends on the coordinates chosen. We attribute the difference in the results to the symmetric coordinates chosen in Ref. [48], which are possibly not well suited to describe the dissociation, especially in an adiabatic approximation. Recently, Villarreal and coworkers performed two-dimensional calculations [52] within an adiabatic approximation, and the simulated absorption spectra show similar peaks, whose widths, calculated within a golden-rule approach, are in semiquantitative agreement with those obtained in this work. In that work [52], the resonances with widths narrower than $1 \mathrm{~cm}^{-1}$ were not considered in the spectrum. Here, however, we include all the peaks since they are obtained at once.

The spectrum is formed by several progressions. In order to assign them, the wave-function amplitudes associated with the most relevant ones are shown in the bottom panel of Fig. 8 . These plots correspond to the wave functions calculated at energies close to the resonances. These wave functions have a bound as well as a dissociative part. Assuming that the bound part is essentially restricted to $\rho<5$ a.u., the nodes can be attributed to the symmetric $(\rho)$ and antisymmetric $(\xi)$ vibrations. We also used for the assignment the progression of the intensity of lines. The final assignment is shown in Fig. 8. Each progression corresponds to a particular antisymmetric mode, characterized by the quantum number $a$. This number is always odd because the ground state is even and the electric dipole $d_{z}$ is odd. The first progression, for $a=1$, corresponds to bound-bound transitions below the dissociation threshold at $3379.6 \mathrm{~cm}^{-1}$. Above the threshold energy, the progressions correspond to $a=3,5$, and 7, with an energy separation among them of about $2500 \mathrm{~cm}^{-1}$. Within each band, the different peaks are attributed to different excitations of the symmetric quantum number $s$. The energy separation between them is of the order of $500-900 \mathrm{~cm}^{-1}$. This assignment is 


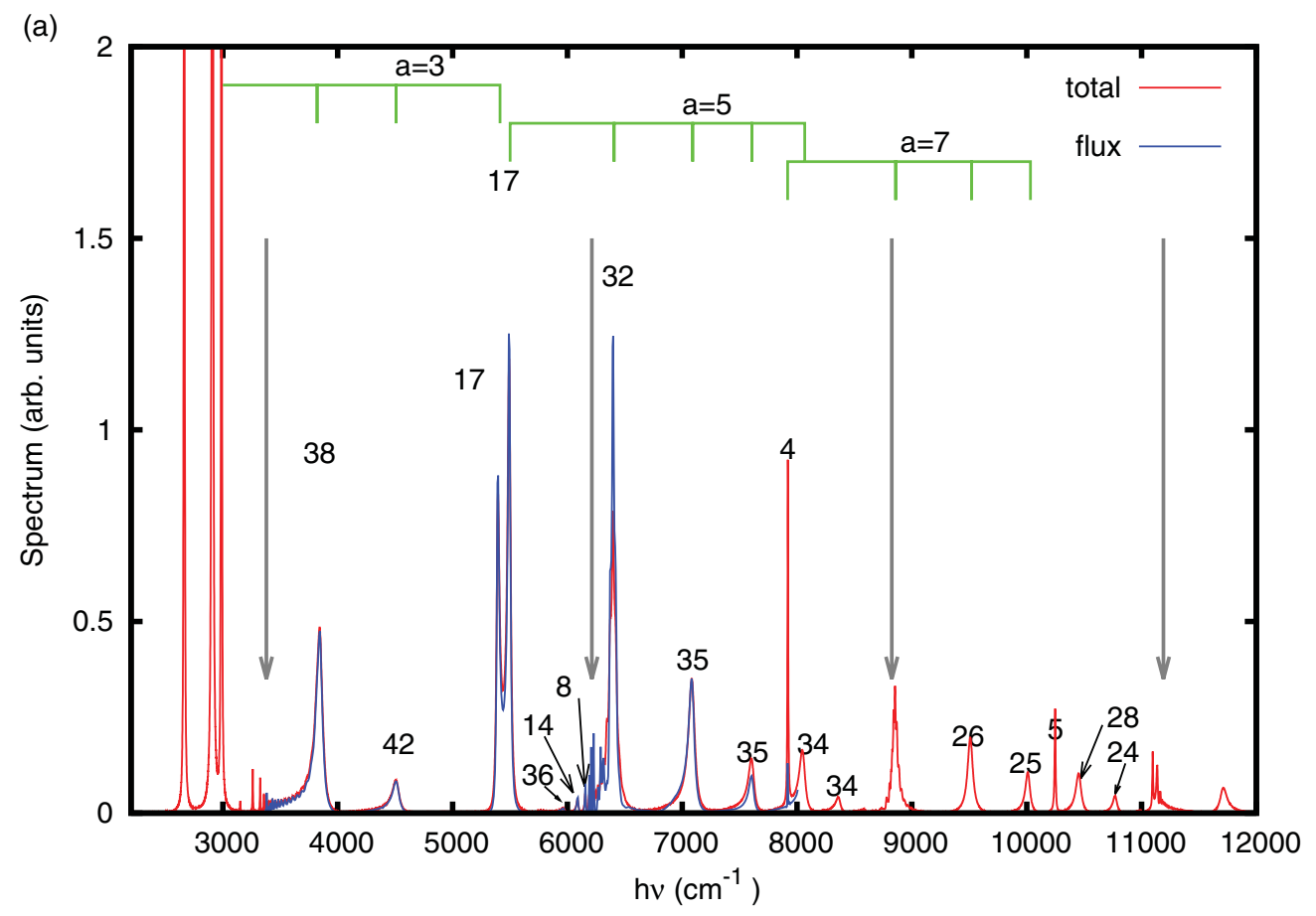

(b)
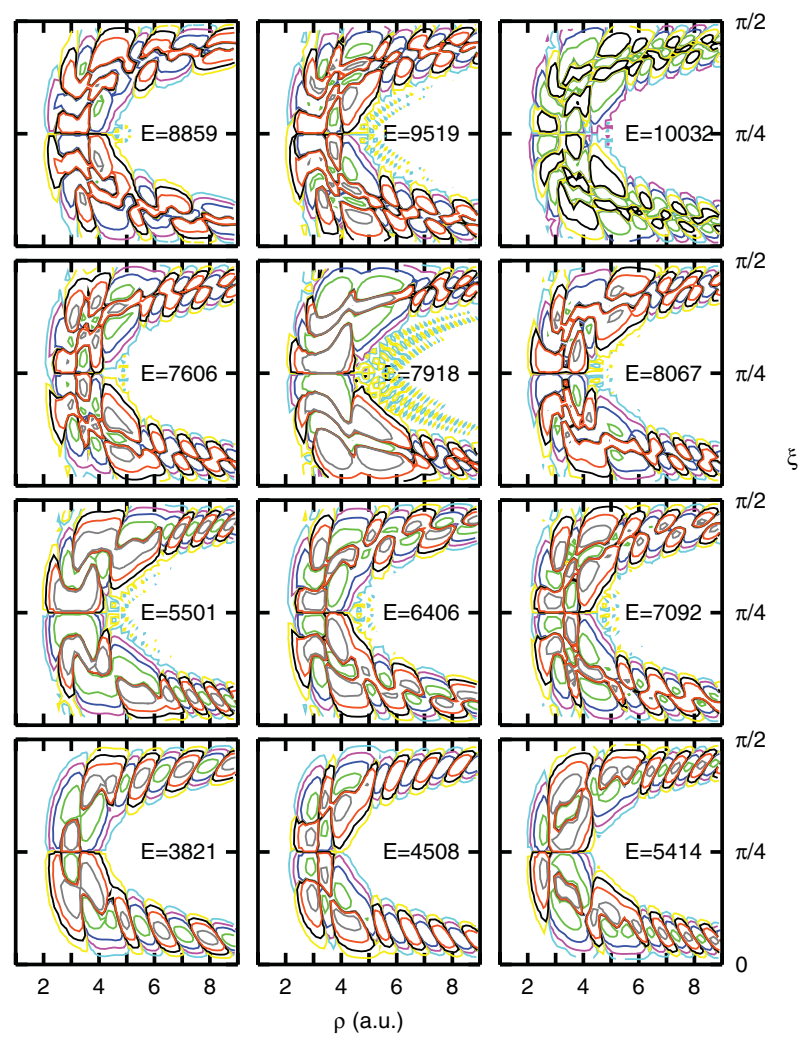

FIG. 8. (Color online) (a) Predissociation spectra for the ground vibrational state as a function of the excitation energy, obtained within the $2 \mathrm{D}$ model using the $d_{z}$ component of the electric dipole, which is the only nonzero component at the nuclear configuration chosen. The numbers appearing close to some peaks are the fitted half widths at half maximum (in $\mathrm{cm}^{-1}$ ). The arrows correspond to the vibrational energies of the $1 \mathrm{D} \mathrm{H}_{3}{ }^{+}$fragments, described by $R_{1}, r_{1}$, and $\theta_{1}$, the last two being frozen in this model. (b) Contour plots of the wave-function amplitudes obtained according to Eq. (14) at the energies indicated, used for the assignment.

completely analogous to that made before in the adiabatic approximation [52]. For example, the progression for $a=3$, formed by the peaks at $\approx 3848$ and 4517 and the doublet at $5451 \mathrm{~cm}^{-1}$, correspond to the $(v, n)=(3,2),(3,3)$, and $(3,4)$ levels of Ref. [52], with energies 4343, 4899, and $5325 \mathrm{~cm}^{-1}$, which are shifted toward higher energies because 
of the adiabatic approximation. The experimental transitions are at 3520,3904 , and $4232 \mathrm{~cm}^{-1}$, shifted between 300 and $1200 \mathrm{~cm}^{-1}$ toward lower energy, showing that something is missing in the simulated spectrum, as will be discussed below. The nonadiabatic effects manifest here also in the presence of the doublet at $\approx 5451 \mathrm{~cm}^{-1}$. The widths of those levels in this work are 38,42 , and $17 \mathrm{~cm}^{-1}$, respectively, while in Ref. [52] they were 54, 58, and $48 \mathrm{~cm}^{-1}$, different because of the golden-rule approximation used there. Nevertheless, there is a semiquantitative agreement between the two sets of widths, except for the third level mentioned, whose width is shared in the doublet appearing because of the nonadiabatic couplings. The energy separation among these levels is of the order of those reported in experiments [22-25], for the energy intervals $2000 \leqslant h v \leqslant 4500$ and $6000 \leqslant h v \leqslant 8000 \mathrm{~cm}^{-1}$. In the second energy intervals the peaks here appear at 6403 , 7053,7617 , and $7969 \mathrm{~cm}^{-1}$ in this work, at 6686, 7272, and $7724 \mathrm{~cm}^{-1}$ in Ref. [52], and the experimental values are 6690, 7130,7490 , and $7770 \mathrm{~cm}^{-1}$ in Ref. [24]. The energy difference between simulated and experimental transitions is of the order of $200 \mathrm{~cm}^{-1}$ but can be used to assign qualitatively the experimental transitions, complementing previous assignments made using the similarity of the peaks measured with the $v_{2}$ frequency of $\mathrm{H}_{3}^{+}$[23-25].

\section{B. Three-dimensional infrared spectrum}

To compare more quantitatively with the experimental results, several deficiencies of the simple $2 \mathrm{D}$ model should be addressed. These problems can be summarized in the abnormally high dissociation energy, $3379.6 \mathrm{~cm}^{-1}$ instead of the $\approx 2400 \mathrm{~cm}^{-1}$ found experimentally, and the fact that $d_{z}$ is the only nonzero component of the electric dipole moment for the equilibrium configuration used in the $2 \mathrm{D}$ model. These two deficiencies are improved when a 3D model with the $R_{1}, R_{2}$, and $\gamma$ coordinates is considered. Within this 3D model, the asymptotic $\mathrm{H}_{3}^{+}$levels are the same as in the 2D model, but the dissociation energy decreases to $2308 \mathrm{~cm}^{-1}$, simply because the ZPE of the ground level increases, as listed in Table II. Also, in the planar configuration used, the $d_{x}$ component is nonzero and contributes to the total spectrum. For the $3 \mathrm{D}$ calculation we considered the small $90 \times 90$ radial grid and 30 Legendre functions for the description of the $\gamma$ angle.

The calculated spectra for perpendicular $\left(d_{x}\right)$ and parallel $\left(d_{z}\right)$ transitions are presented in the two top panels of Fig. 9, in the same units, and compared with the experimental spectrum of Ref. [25]. The perpendicular spectrum $d_{x}$ has lower intensity than the $d_{z}$ one, simply because the electric dipole moment varies more rapidly along the $z$ axis, where the proton exchange between the two $\mathrm{H}_{2}$ molecules is produced. Since along this motion the charges vary greatly, the electric dipole also does, and it is the $z$ component which carries most of the oscillator strength.

The spectrum obtained for the parallel transition presents a well-known similarity with the experimental one, especially when considering the simplicity of the model. The first experimental peak, appearing at $2603 \mathrm{~cm}^{-1}$, is transformed into an intense band formed by very narrow peaks in the simulation. These structures appear very close to the dissociation threshold, $2408 \mathrm{~cm}^{-1}$ in this case. The simulated higher-energy peaks show a better agreement with respect to the experimental one, in position and intensity, and even the widths. The experimental peak at $3520 \mathrm{~cm}^{-1}$ is shifted to $3476 \mathrm{~cm}^{-1}$ in the simulations, only $44 \mathrm{~cm}^{-1}$. The other two peaks at $4232(4154)$ and $3904(4003) \mathrm{cm}^{-1}$ in the experiments (simulations) are also in quite good agreement, with a difference of 78 and $99 \mathrm{~cm}^{-1}$. Thus the inclusion of the angle $\gamma$ improves considerably the agreement between simulated and experimental spectra with respect to the $2 \mathrm{D}$ model discussed above. It simply seems that the intensities of these two peaks are interchanged, possibly because of the reduced-dimensionality model used.

The isolated peaks of the spectrum have been fitted to a Lorentzian function, and their widths are given in the spectrum in Fig. 9. They are slightly narrower than in the 2D case, indicating that the dissociation is less efficient. The excitation of the $\gamma$ angle somehow deviates energy from the vibrational motions, which are the ones that yield to dissociation. Some of the resonances may be even narrower, since the time window used introduces a broadening of $\approx 3 \mathrm{~cm}^{-1}$ in the bound-bound transitions when using Eq. (12). In this regard, it should be noted that the peaks appearing below $3000 \mathrm{~cm}^{-1}$ are very narrow. This means that the simulated peaks present a much higher intensity, while the integration in energy should somehow reduce it.

The wave functions associated with the resonances present higher complexity than the 2D ones, and the modes are more mixed. When the bound part is associated with $\rho<5$ a.u. in the lower panels of Fig. 9, the radial nodal structure is not as clean as in the 2D case, but still most of the resonances can be attributed to three quanta in the asymmetric stretch $a=3$. The structure in the angular coordinate $\gamma$ is much more complex and complicated to assign, and is distributed over the whole angular interval. This motion is associated with the scrambling mechanism, since it would allow the central hydrogen to be exchanged with any lateral one if all the dimensions were taken into account, as illustrated in Fig. 6. However in the present reduced-dimensionality model, the different minima are not described symmetrically and hence the corresponding peaks should be affected.

The 3D model applied to $\mathrm{H}_{5}{ }^{+}$seems to be rather realistic, in spite of its simplicity, and allows the approximate assignment of the observed transitions in the spectrum. The major change in the electric dipole corresponds to the proton exchange between the two $\mathrm{H}_{2}$ subunits, and it is the origin of the observed vibrational progressions assigned in the simulated spectrum. To check this model, it has also been applied to $\mathrm{D}_{5}{ }^{+}$, whose simulated spectrum is compared with the experimental one in Fig. 10. The simulated spectrum is composed of three bands of narrow peaks. Due to the increase of the mass, the wave functions are more localized in the well and the rates for dissociation smaller, producing a narrowing of the absorption lines as compared with $\mathrm{H}_{5}{ }^{+}$. In this case the difference between experimental and simulated widths is larger than for $\mathrm{H}_{5}{ }^{+}$. The widths in both systems seem to decrease when the number of degrees of freedom is increased. Part of the disagreement is attributed to the rotational broadening, not included in the present model.

The relative intensities are in an good approximate agreement with the observed ones, although the positions are not 

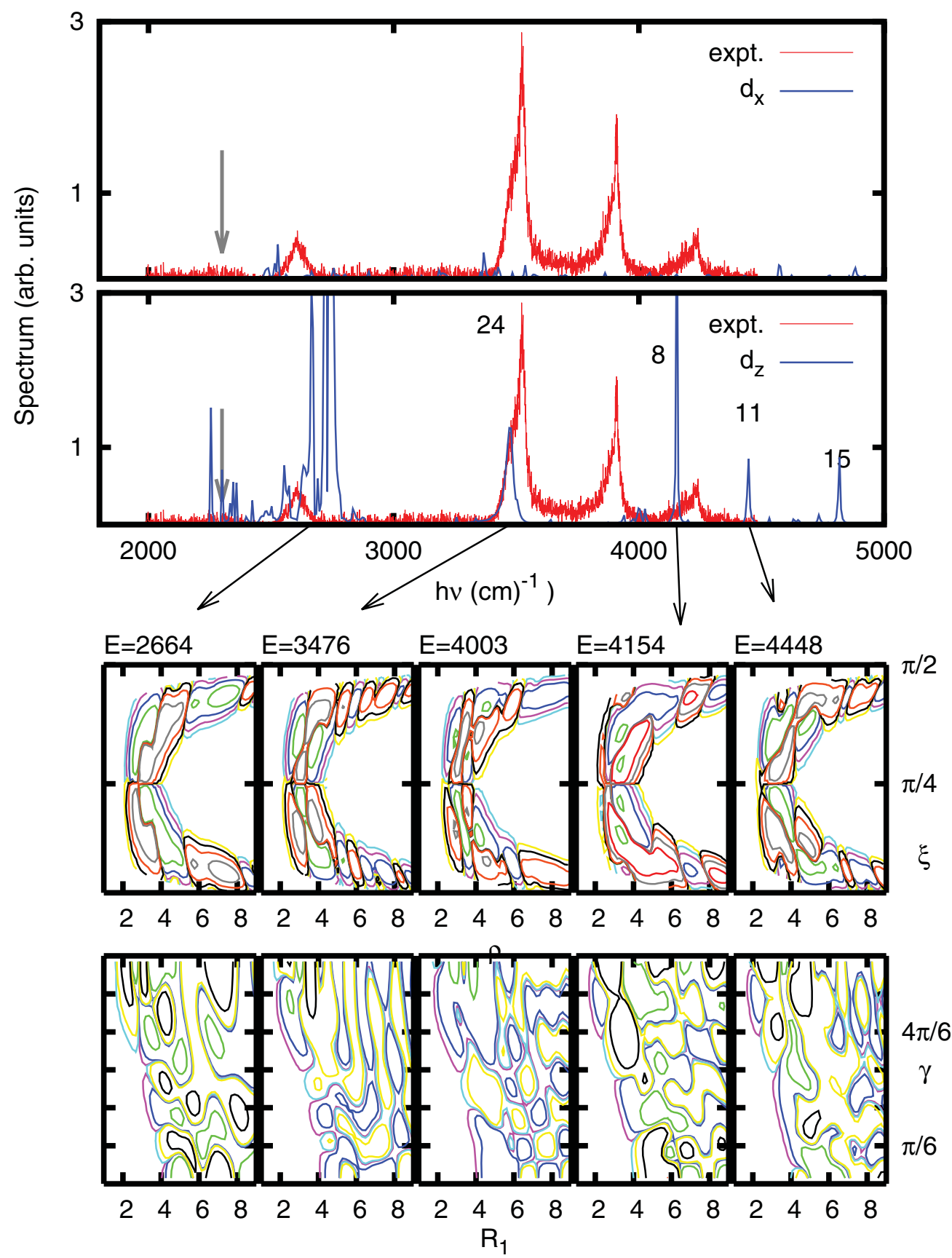

FIG. 9. (Color online) Predissociation spectra for the ground vibrational state as a function of the excitation energy, obtained within the 3D model using the $d_{x}$ (top panel) and $d_{z}$ (second panel from the top) components of the electric dipole. The experimental data are taken from Ref. [25] and shown in arbitrary units. The numbers appearing close to some peaks are the fitted half widths at half maximum (in $\mathrm{cm}^{-1}$ ). The arrows correspond to the vibrational energies of the $1 \mathrm{D} \mathrm{H}_{3}{ }^{+}$fragments, as in Fig. 8. The bottom panels are the contour plots of the amplitudes of the wave functions obtained according to Eq. (14) at the energies indicated, as a function of $R_{1}, \gamma$ with $R_{2}=1.6$ a.u. (bottom panels), $\rho=\sqrt{R_{1}^{2}+R_{2}^{2}}$, and $\xi=\arctan \left(R_{1} / R_{2}\right)$ with $\gamma=\pi$.

as good. The first band is shifted toward lower energies, while the other two are shifted toward higher energies. Thus the simulated energy separation between peaks is larger than in the experimental bands. This effect appears also in the spectrum of $\mathrm{H}_{5}^{+}$, but for $\mathrm{D}_{5}^{+}$it is more noticeable. Nevertheless, it should be noted that the experimental spectrum of $\mathrm{D}_{5}{ }^{+}$becomes nearly zero for frequencies larger than $\approx 3200 \mathrm{~cm}^{-1}$, what is not the case in the simulated one. As studied in more detail for $\mathrm{H}_{5}{ }^{+}$, there are more absorption bands as the energy increases, of decreasing intensity. However, the complete suppression of the observed intensity is not understood.

The reason for many of the simulated vs experimental differences may be associated with the reduced-dimensionality model used, which yields lower ZPEs. The inclusion of more degrees of freedom is difficult, not only because of the increase of the dimensionality of the problem but also because the computational time increases. Several attempts have been made including more degrees of freedom, not completely 


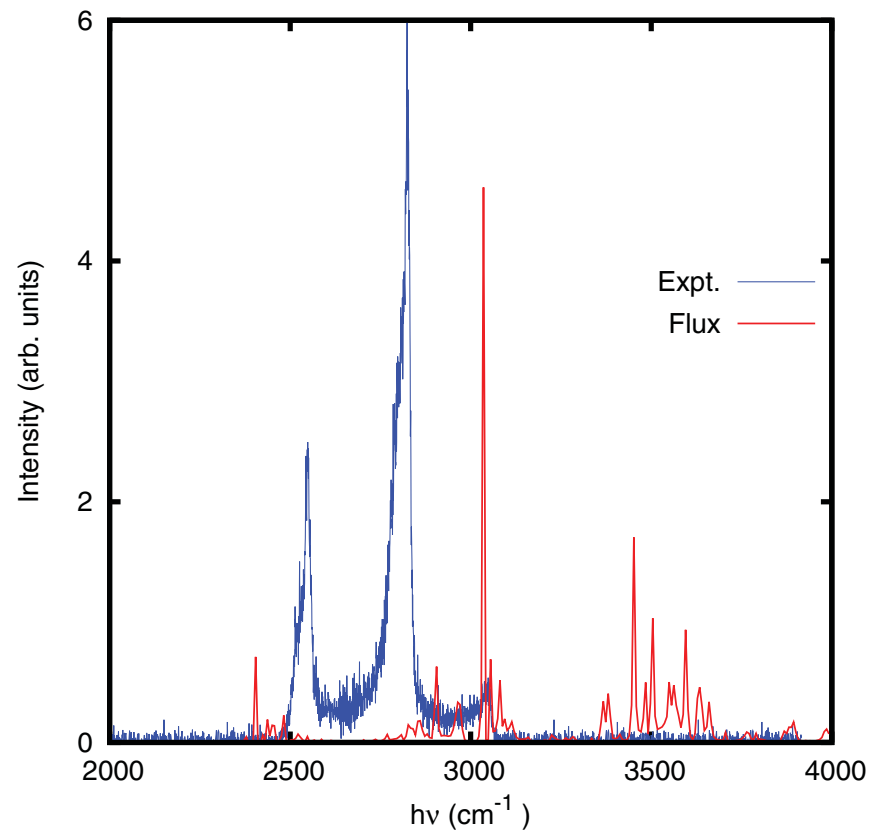

FIG. 10. (Color online) Predissociation spectra for the ground vibrational state as a function of the excitation energy, as in Fig. 9, but for $\mathrm{D}_{5}^{+}$. The experimental data are taken from Ref. [25] and shown in arbitrary units.

converged, and the high intense peaks near the dissociation threshold are always there.

These near-threshold resonances may have an important role in the ortho-to-para conversion of $\mathrm{H}_{3}{ }^{+}$and its deuteration in collisions. Until now only statistical studies of these processes have been performed [11,12,20]. In these studies two limiting models are assumed: a complete scrambling in which an exchange of any proton is produced, or a restricted-protonhop mechanism, in which the direct proton transfer from the $\mathrm{H}_{3}^{+}$to the $\mathrm{H}_{2}$ subunit becomes more favorable. These two processes are going to be extremely sensitive to the nature and widths of the resonances involved. At the low temperatures of the interstellar medium, $\approx 10 \mathrm{~K}$, the near-threshold resonances are important. In this regard, the spectroscopic study of these resonances may provide important information about them. Their experimental study is not absent of difficulties, and as an example, the laser power used in the experiments of Ref. [25] is five times greater in the higher-frequency range than at the lower end [86]. In addition, in the model 2D study performed recently [52], the narrower resonances were removed, yielding a reasonable qualitative agreement with the experimental results. Thus the question of whether extremely narrow resonances may present difficulties for experimental observation should be investigated.

Nevertheless, the main problem is on the theoretical side, where a much deeper effort must still be made, due to the difficulty of a complete quantum study on this system. To describe more accurately the whole system, we need to take into account all possible configuration minima, which requires the inclusion of all degrees of freedom. Otherwise, the minima are not perfectly equivalent due to the restrictions made with these coordinates. As an example, in Fig. 6 two of these scrambling minima are present, and since $\gamma$ varies from 0 to $\pi$, only atoms 0 and 2 can be central atoms, while 1 cannot because it would require $\gamma>\pi$. Also, the inclusion of the remaining degrees of freedom will increase the ZPEs. Finally, when considering the motion of all the $\mathrm{H}$ atoms, we should also account for the symmetry of the whole system.

In this regard, it should be mentioned that recently a simulation of the spectra of these systems have been performed using the multimode reaction path method [25], including all vibrational degrees of freedom for zero total angular momentum. The agreement with the experiment is not better than that obtained here in spite of the full dimensionality, probably for two reasons. First, the method is based on a normal-mode expansion, which may not be suited for describing large-amplitude motions, and fragmentation, important in this system. Second, the spectra are simulated as if they correspond to bound-bound transitions, while in reality the excited spectra correspond to resonances. All this indicates that more effort should be made to study the predissociation dynamics of such complicated symmetric and quantum systems.

\section{CONCLUSIONS}

In this work the predissociation spectrum of $\mathrm{H}_{5}^{+}$has been studied. For this purpose, the electric dipole moment has been calculated using a CCSD method at about 100000 points and fitted to an analytical form. This fit is based on a TRIM or DIM model, as previously used for the PES [40], which compares nicely with the ab initio results and provides a rather simple physical interpretation of the $\mathrm{H}_{3}{ }^{+}+\mathrm{H}_{2} \rightarrow \mathrm{H}_{2}+\mathrm{H}_{3}{ }^{+}$ charge-transfer process.

The bound states of $\mathrm{H}_{5}^{+}$have been calculated using an iterative Lanczos method, including from two to seven degrees of freedom. The 7D zero-point energy thus obtained is $7167 \mathrm{~cm}^{-1}$, in good agreement with results of previous calculations on different PESs. Considering the zero-point energies of the $\mathrm{H}_{2}$ and $\mathrm{H}_{3}^{+}$fragments and using also the PES of Ref. [40], a dissociation energy of $2415 \mathrm{~cm}^{-1}$ is obtained, in good agreement with previous theoretical $[25,39,81,82]$ and experimental [83-85] results.

To describe the $\mathrm{H}_{3}^{+}+\mathrm{H}_{2}$ dissociation, a wave-packet treatment has been employed, while until now most of the theoretical studies were based on bound-bound transition models $[25,36,46,75]$, which do not incorporate the dissociation dynamics. The predissociation spectra have been calculated using 2D and 3D reduced-dimensional simulations based on a proton-share model [52]. This model is based on considering that the electric dipole varies mainly due to proton transfer between the two $\mathrm{H}_{2}$ units, described by the $R_{1}, R_{2}$, and $\gamma$ coordinates. It is found that the main contribution arises from parallel transitions associated with the proton-exchange process. The main simulated peaks are in reasonably good agreement with the peaks in the experimental spectrum [25], in the 3D case. More degrees of freedom should also be considered to reach a better agreement with the experimental spectrum. Also, some experimental conditions should be taken into account to improve the comparison, such as the dependence of the laser power on its wavelength, which in Ref. [25] is five times greater in the higher-frequency range than at the lower end [86]. 
These models are approximate with respect to the experimental results, but allow a semiquantitative assignment. Also, they provide a good physical interpretation of the spectra: since the electric dipole moment has a weak dependence on the remaining degrees of freedom, they are not excited and can be considered as spectators in the absorption process. Thus, the detected lines are scarcely related to the $v_{2}$ transition of $\mathrm{H}_{3}^{+}$, since the PES is modified enormously when the other $\mathrm{H}_{2}$ molecule approaches. Here, the spectrum is assigned to an overtone of the antisymmetric stretch of the proton, $a=3$, which carries most of the oscillator strength for the infrared transition. Thus, the three experimental peaks would correspond to different symmetric stretching quantum numbers $s$ and $a=3$.

The linewidths have been determined, varying from 1 to $50 \mathrm{~cm}^{-1}$ in the 2D model and from 1 to $10 \mathrm{~cm}^{-1}$ in the 3D model. This decrease of the widths is due to the increase of the number of degrees of freedom, allowing the energy to flow toward nondissociative coordinates. Also, the lines calculated for $\mathrm{D}_{5}{ }^{+}$are considerably narrower than for $\mathrm{H}_{5}{ }^{+}$, as expected. The widths affect the height of the peaks because the intensity corresponds to integration over the energy interval covered by them. The inclusion of the predissociation process is therefore important to get the proper intensity ratios in the absorption spectra.

The inclusion of all the internal degrees of freedom is now being investigated by using computationally more efficient techniques, such as multi-configuration time-dependent Hartree [87]. The use of coordinates better adapted to treat the high symmetry of the system, such as the hyperspherical formalism recently developed by Kuppermann [43,45], is also envisaged.

\section{ACKNOWLEDGMENTS}

We acknowledge Professor M. A. Duncan for providing us with experimental results, and for interesting discussions. This work has been supported by the program CONSOLIDERINGENIO of Ministerio de Ciencia e Innovación under Grant No. CSD2009-00038, entitled "Molecular Astrophysics: the Herschel and Alma Era," and by Grants No. FIS2010-18132 and No. FIS2011-29596-C02, and by Comunidad Autónoma de Madrid (CAM) under Grant No. S-2009/MAT/1467. Also support from European COST Action CODECS (Ref. No. CM1002) is acknowledged. The calculations have been performed at CESGA and IFF computing centers, for which we acknowledge ICS computing grants.
[1] T. J. Millar, A. Bernett, and E. Herbst, Astrophys. J. 340, 906 (1989).

[2] L. Pagani, M. Salez, and P. G. Wannier, Astron. Astrophys. 258, 479 (1992).

[3] J. Tennyson, Rep. Prog. Phys. 58, 412 (1995).

[4] B. J. McCall and T. Oka, Science 287, 1941 (2000).

[5] O. Witasse, T. Cravens, M. Mendillo, J. Mosesand A. Kliore, A. F. Nagy, and T. Breusx, Space Sci. Rev. 139, 235 (2008).

[6] D. L. Huestis, S. W. Bougher, J. L. Fox, M. Galand, R. E. Johnson, J. I. Moses, and J. C. Pickering, Space Sci. Rev. 139, 63 (2008).

[7] L. E. Moore, M. Mendillo, I. C. F. Müller-Wodarg, and D. L. Murr, Icarus 172, 503 (2004).

[8] M. Jiménez-Redondo, E. Carrasco, V. J. Herrero, and I. Tanarro, Phys. Chem. Chem. Phys. 13, 9655 (2011).

[9] M. Quack, Mol. Phys. 34, 21 (1977).

[10] T. Oka, J. Mol. Spectrosc. 228, 635 (2004).

[11] D. Gerlich, F. Windisch, P. Hlavenka, R. Plasil, and J. Glosik, Philos. Trans. R. Soc., A 364, 3007 (2006).

[12] K. Park and J. C. Light, J. Chem. Phys. 126, 044305 (2007).

[13] D. Uy, M. Cordonnier, and T. Oka, Phys. Rev. Lett. 78, 3844 (1997).

[14] T. J. Millar, Planet. Space Sci. 50, 1189 (2002).

[15] D. Gerlich, J. Chem. Phys. 92, 1141 (1990).

[16] D. Gelich and S. Schlemmer, Planet. Space Sci. 50, 1287 (2002).

[17] D. Smith, N. G. Adams, and E. Alge, Astrophys. J. 263, 123 (1982).

[18] K. Giles, N. G. Adams, and D. Smith, J. Phys. Chem. 96, 7645 (1992).

[19] D. Gerlich, E. Herbst, and E. Roueff, Planet. Space Sci. 50, 1275 (2002).
[20] E. Hugo, O. Asvany, and S. Schlemmer, J. Chem. Phys. 130, 164302 (2009).

[21] K. N. Crabtree, C. A. Kauffman, B. A. Tom, E. Becka, B. A. McGuire, and B. J. McCall, J. Chem. Phys. 134, 194311 (2011).

[22] M. Okumura, L. I. Yeh, and Y. T. Lee, J. Chem. Phys. 83, 3705 (1985).

[23] M. Okumura, L. I. Yeh, and Y. T. Lee, J. Chem. Phys. 88, 79 (1988).

[24] Y. K. Bae, Chem. Phys. Lett. 180, 179 (1991).

[25] T. C. Cheng, B. Bandyopadyay, Y. Wang, S. Carter, B. J. Brraams, J. M. Bowman, and M. A. Duncan, J. Phys. Chem. Lett. 1, 758 (2010).

[26] Y. Yamaguchi, J. F. Gaw, B. Remington, and H. F. Shaefer III, J. Chem. Phys. 86, 5072 (1987).

[27] M. Farison, H. Chermentte, and B. Farison-Mazuy, J. Chem. Phys. 96, 1325 (1992).

[28] T. Pang, Chem. Phys. Lett. 228, 555 (1994).

[29] I. Štich, D. Marx, M. Parrinello, and K. Terakura, J. Chem. Phys. 107, 9482 (1997).

[30] E. W. Ignacio and S. Yamabe, Chem. Phys. Lett. 287, 563 (1998).

[31] H. Müller and W. Kutzelnigg, Phys. Chem. Chem. Phys. 2, 2061 (2000).

[32] M. Barbatti, G. Jalbert, and M. A. C. Nascimento, J. Chem. Phys. 113, 4230 (2000).

[33] M. Barbatti, G. Jalbert, and M. A. C. Nascimento, J. Chem. Phys. 114, 7066 (2001).

[34] R. Prosmiti, P. Villarreal, and G. Delgado-Barrio, J. Phys. Chem. A 107, 4768 (2003).

[35] U. Nagashima, K. Morokuma, and H. Tanaka, J. Phys. Chem. 96, 4294 (1992).

[36] W. P. Kraemer, V. Spirko, and O. Bludský, J. Mol. Spectrosc. 164, 500 (1994). 
[37] R. Prosmiti, A. A. Buchachenko, P. Villarreal, and G. DelgadoBarrio, Theor. Chem. Acc. 106, 426 (2001).

[38] G. E. Moyano and M. A. Collins, J. Chem. Phys. 119, 5510 (2003).

[39] Z. Xie, B. J. Braams, and J. M. Bowman, J. Chem. Phys. 122, 224307 (2005).

[40] A. Aguado, P. Barragán, R. Prosmiti, G. Delgado-Barrio, P. Villarreal, and O. Roncero, J. Chem. Phys. 133, 024306 (2010). The program with the PES is available at [http://fama.iff.csic.es/personas/octavio/metodos-programas/ PES/PES.html].

[41] P. Barragan, R. Prosmiti, O. Roncero, A. Aguado, P. Villarreal, and G. Delgado-Barrio, J. Chem. Phys. 133, 054303 (2010).

[42] M. Cordonnier, D. Uy, R. M. Dickson, K. E. Kerr, Y. Zhang, and T. Oka, J. Chem. Phys. 113, 3181 (2000).

[43] A. Kuppermann, J. Phys. Chem. A 113, 4518 (2009).

[44] A. Kuppermann (private communication).

[45] A. Kuppermann, Phys. Chem. Chem. Phys. 13, 8259 (2011).

[46] P. Soldan, J. Mol. Spectros. 168, 258 (1994).

[47] P. Soldan, V. Spirko, and W. P. Kraemer, J. Mol. Spectrosc. 183, 212 (1997).

[48] V. Spirko, T. Amano, and W. P. Kraemer, J. Chem. Phys. 124, 244303 (2006).

[49] D. Wang, Z. Xie, and J. M. Bowman, J. Chem. Phys. 132, 084305 (2010).

[50] S. Carter, S. J. Culik, and J. M. Bowman, J. Chem. Phys. 107, 10458 (1997).

[51] D. P. Tew, C. Handy, S. Carter, S. Irle, and J. M. Bowman, Mol. Phys. 101, 3513 (2003).

[52] C. Sanz-Sanz, O. Roncero, A. Valdes, R. Prosmiti, G. DelgadoBarrio, P. Villarreal, P. Barragan, and A. Aguado, Phys. Rev. A 84, 060502 (2011).

[53] P. Villarreal, O. Roncero, and G. Delgado-Barrio, J. Chem. Phys. 101, 2217 (1994).

[54] M. Lara, A. Aguado, M. Paniagua, and O. Roncero, J. Chem. Phys. 113, 1781 (2000).

[55] R. N. Zare, Angular Momentum (John Wiley and Sons, New York, 1988).

[56] J. T. Muckerman, Chem. Phys. Lett. 173, 200 (1990).

[57] J. C. Light, I. P. Hamilton, and J. V. Lill, J. Chem. Phys. 82, 1400 (1985).

[58] G. C. Corey, J. W. Tromp, and D. Lemoine, in Numerical Grid Methods and Their Application to the Schrödinger Equation, edited by C. Cerjan (Kluwer Academic, Dordrecht, 1993), p. 1.

[59] C. Leforestier, J. Chem. Phys. 101, 7357 (1994).

[60] O. Roncero, D. Caloto, K. C. Janda, and N. Halberstadt, J. Chem. Phys. 107, 1406 (1997).

[61] C. Lanczos, J. Res. Natl. Bur. Stand. 45, 255 (1950).

[62] J. K. Cullum and R. A. Willoughby, Lanczos Algorithms for Large Symmetric Eigenvalue Computations (Birkhäuser, Boston, 1985).

[63] C.-E. Fröberg, Numerical Mathematics: Theory and Computer Applications (Benjamin/Cummings, San Francisco, 1985).
[64] R. E. Wyatt, Adv. Chem. Phys. 73, 231 (1989).

[65] Y. Huang, D. J. Kouri, and D. K. Hoffman, J. Chem. Phys. 101, 10493 (1994).

[66] V. A. Mandelshtam and H. S. Taylor, J. Chem. Phys. 103, 2903 (1995).

[67] Y. Huang, S. S. Iyengar, D. J. Kouri, and D. K. Hoffman, J. Chem. Phys. 105, 927 (1996).

[68] G. J. Kroes and D. Neuhauser, J. Chem. Phys. 105, 8690 (1996).

[69] R. Chen and H. Guo, J. Chem. Phys. 105, 3569 (1996).

[70] S. K. Gray and G. G. Balint-Kurti, J. Chem. Phys. 108, 950 (1998).

[71] T. González-Lezana, A. Aguado, M. Paniagua, and O. Roncero, J. Chem. Phys. 123, 194309 (2005).

[72] R. Sadeghi and R. T. Skodje, J. Chem. Phys. 102, 193 (1995).

[73] C. L. Russell and D. E. Manolopoulos, Chem. Phys. Lett. 256, 465 (1996).

[74] M. Paniagua, A. Aguado, M. Lara, and O. Roncero, J. Chem. Phys. 109, 2971 (1998); 111, 6712 (1999).

[75] V. Spirko, W. P. Kraemer, and P. Soldan, J. Mol. Spectrosc. 183, 218 (1997).

[76] B. Jin, H. L. Schmider, and D. Wardlaw, Chem. Phys. Lett. 317, 464 (2000).

[77] MOLPRO is a package of ab initio programs designed by H.-J. Werner and P. J. Knowles and with contributions from J. Almlöf, R. D. Amos, A. Berning, M. J. O. Deegan, F. Eckert, S. T. Elbert, C. Hampel, R. Lindh, W. Meyer, A. Nicklass, K. Peterson, R. Pitzer, A. J. Stone, P. R. Taylor, M. E. Mura, P. Pulay, M. Schütz, H. Stoll, T. Thorsteinsson, and D. L. Cooper (2006).

[78] T. H. Dunning Jr., J. Chem. Phys. 90, 1007 (1989).

[79] A. Aguado, O. Roncero, C. Tablero, C. Sanz, and M. Paniagua, J. Chem. Phys. 112, 1240 (2000).

[80] C. Sanz. O. Roncero, C. Tablero, A. Aguado, and M. Paniagua, J. Chem. Phys. 114, 2182 (2001).

[81] R. Perez de Tudela, P. Barragán, R. Prosmiti, P. Villarreal, and G. Delgado-Barrio, J. Phys. Chem. A 115, 2483 (2011).

[82] P. H. Acioli, Z. Xie, B. J. Braamas, and J. M. Bowman, J. Chem. Phys. 128, 104318 (2008).

[83] R. J. Beuhler, S. Ehrenson, and L. Friedman, J. Chem. Phys. 79, 5982 (1983).

[84] K. Hiraoka, J. Chem. Phys. 87, 4048 (1987).

[85] K. Hiraoka and T. Mori, J. Chem. Phys. 91, 4821 (1989).

[86] M. A. Duncan (private communication).

[87] M. H. Beck, A. Jäckle, G. A. Worth, and H.-D. Meyer, Phys. Rep. 324, 1 (2000).

[88] R. Prosmiti, O. L. Polyansky, and J. Tennyson, Chem. Phys. Lett. 273, 107 (1997).

[89] R. Jaquet, W. Cencek, W. Kutzelnigg, and J. Rychlewski, J. Chem. Phys. 108, 2837 (1998).

[90] B. M. Dinelli, L. Neale, O. L. Polyansky, and J. Tennyson, J. Mol. Spectrosc. 181, 142 (1997). 